\title{
THE WREATH PRODUCT OF ATOMS OF THE LATTICE OF SEMIGROUP VARIETIES
}

\author{
A. V. TISHCHENKO
}

\begin{abstract}
A semigroup variety is called a Cross variety if it is finitely based, is generated by a finite semigroup, and has a finite lattice of subvarieties. It is established in which cases the wreath product of two semigroup varieties each of which is an atom of the lattice of semigroup varieties is a Cross variety. Furthermore, for all the pairs of atoms $\mathbf{U}$ and $\mathbf{V}$ for which this is possible, either a finite basis of identities for the wreath product $\mathbf{U w} \mathbf{V}$ is given explicitly, a finite semigroup generating this variety is found and the lattice of subvarieties is described, or it is proved that such a finite characterization is impossible.
\end{abstract}

\section{Statement of the PROBlem And Formulation of the MAin RESUlts}

This paper is devoted to a systematic study of the wreath products of atoms of the lattice of semigroup varieties. We consider three classical conditions for varieties to be finite: having a finite basis of identities, being generated by a finite semigroup, and having a finite lattice of subvarieties. For all the pairs of atoms $\mathbf{U}$ and $\mathbf{V}$ for which this is possible, a finite basis of identities for the wreath product $\mathbf{U} \mathbf{w} \mathbf{V}$ is given explicitly, a finite semigroup generating this variety is found and the lattice of subvarieties is described.

Definition 1.1. A semigroup variety is called a Cross variety if it is finitely based, is generated by a finite semigroup and has a finite lattice of subvarieties.

The atoms of the lattice $\mathcal{L}$ of all semigroup varieties are well known [16]. These are precisely the varieties $\mathbf{N}_{\mathbf{2}}$ of all semigroups with zero multiplication, $\mathbf{S l}$ of all semilattices, $\mathbf{L}_{\mathbf{1}}$ of all semigroups of left zeros, $\mathbf{R}_{\mathbf{1}}$ of all semigroups of right zeros and $\mathbf{A}_{\mathbf{p}}$ of all Abelian groups of prime exponent $p$. The main result of the paper can be stated as follows.

Theorem 1.2. If $\mathbf{U}$ and $\mathbf{V}$ are atoms of the lattice of semigroup varieties, then the wreath product $\mathbf{U w} \mathbf{V}$ is a Cross variety, except in the following cases:

1) $\mathbf{U}=\mathbf{V}=\mathbf{A}_{\mathbf{p}}$; here the variety $\mathbf{A}_{\mathbf{p}} \mathbf{w} \mathbf{A}_{\mathbf{p}}=\mathbf{A}_{\mathbf{p}}^{\mathbf{2}}$ is finitely based but is not generated by a finite semigroup and has an infinite lattice of subvarieties;

2) $\mathbf{U}=\mathbf{V}=\mathbf{S l}$ and $\mathbf{U}=\mathbf{S l}, \mathbf{V}=\mathbf{R}_{\mathbf{1}}$; here each of the varieties $\mathbf{S l w} \mathbf{S l}=\mathbf{S l}^{\mathbf{2}}$ and $\mathbf{S l w R}_{\mathbf{1}}$ is finitely based, is generated by a finite semigroup and has an infinite lattice of subvarieties;

3) $\mathbf{U}=\mathbf{S l}, \mathbf{V}=\mathbf{A}_{\mathbf{p}}$; here the variety $\mathbf{S l w} \mathbf{A}_{\mathbf{p}}$ is essentially infinitely based, is generated by a finite semigroup and has an infinite lattice of subvarieties.

As a by-product, it becomes possible to estimate how big the difference is between the monoid wreath product and the lattice join of two semigroup varieties in the case where the varieties involved in the wreath product are atoms in the lattice of semigroup

2000 Mathematics Subject Classification. Primary 20M07; Secondary $20 \mathrm{E} 22$.

Key words and phrases. Semigroup, atom, lattice of subvarieties, semigroup variety, wreath product, basis of identities, Cross variety. 
varieties. To measure this difference we choose the cardinality of the lattice interval $\mathcal{I}(\mathbf{U}, \mathbf{V})=[\mathbf{U} \vee \mathbf{V}, \mathbf{U w} \mathbf{V}]$ that contains all the varieties between the lattice join and the monoid wreath product of two semigroup varieties that are atoms. As a rule, when this interval is finite, it contains at most 4 elements.

The operation given by a semidirect product of pseudovarieties of monoids and semigroups has been studied fairly intensively since its introduction in Eilenberg's book 23 . in 1976. This interest was caused by Eilenberg's discovery of a one-to-one correspondence between streams of formal rational languages and pseudovarieties of finite monoids (see also [5. Ch. 6, Theorem 5.11]). Here we study the operation given by a monoid wreath product of semigroup varieties (see [33, 9]). In this paper we attempt to give a quantitative estimate of this operation based on the example of the wreath product of atoms of the lattice $\mathcal{L}$ of semigroup varieties. We mention that the monoid of semigroup varieties with the operation of monoid wreath product was studied in [11, 15].

Just by using the example of atoms of the lattice $\mathcal{L}$ one can see that for multiplication (taking the monoid wreath product) on the left by the atoms $\mathbf{N}_{\mathbf{2}}, \mathbf{L}_{\mathbf{1}}, \mathbf{R}_{\mathbf{1}}$ the operation is small and the cardinality of the interval $\mathcal{I}(\mathbf{U}, \mathbf{V})$ does not exceed 3 . This is also almost always the case for multiplication on the left by a group atom, apart from $\mathbf{A}_{\mathbf{p}}^{2}$. In the latter case, the lattice $\mathcal{L}\left(\mathbf{A}_{\mathbf{p}}^{\mathbf{2}}\right)$ and the corresponding interval are infinite. In the case of two different group atoms, it is known from Higman's theorem [6] that the cardinality of the lattice $\mathcal{L}(\mathbf{U w} \mathbf{V})$ is equal to 5 . But the worst behaviour is that of the operation for multiplication (taking the monoid wreath product) on the left by the atom $\mathbf{S l}$ of all semilattices. Here, as a rule, the interval $\mathcal{I}(\mathbf{U}, \mathbf{V})$ under consideration is infinite, apart from the monoid wreath products $\mathbf{S l w N}_{2}$ and $\mathbf{S l w} \mathbf{L}_{1}$. Furthermore, although in the case of $\mathbf{S l w} \mathbf{N}_{\mathbf{2}}$ the lattice $\mathcal{L}\left(\mathbf{S l w} \mathbf{N}_{2}\right)$ is finite, it is fairly large, namely, it contains at least 33 subvarieties. Also, although the interval $\mathcal{I}\left(\mathbf{S l}, \mathbf{N}_{\mathbf{2}}\right)$ is finite, it contains at least 18 varieties. We point out that in many cases we succeeded in calculating the lattices $\mathcal{L}(\mathbf{U}, \mathbf{V})$ for atoms $\mathbf{U}$ and $\mathbf{V}$ explicitly.

First, a basis of identities and, in certain cases, a finite semigroup is calculated for the wreath product of atoms $\mathbf{U}$ and $\mathbf{V}$ of the lattice $\mathcal{L}$ that generates this wreath product. In calculating the bases of identities the results of [10] play an essential role. (See also [12.) Next, for all atoms $\mathbf{U}$ and $\mathbf{V}$, the lattices $\mathcal{L}(\mathbf{U w} \mathbf{V})$ of all subvarieties of the wreath products $\mathbf{U w} \mathbf{V}$ are calculated, and then the lattice intervals $\mathcal{I}(\mathbf{U}, \mathbf{V})$ are calculated. In 24] it is noted that if a finite semigroup has a finite basis of identities, then the same basis of identities is a basis of the pseudovariety generated by this finite semigroup. On the other hand, Theorem 4.1 shows that in almost all the cases under consideration, the wreath product of atoms of varieties is generated by a finite semigroup. These two facts show that the results obtained are important for pseudovarieties of finite semigroups.

Remark 1.3. The fact that the wreath product of two group varieties has a finite basis follows from the results of the well-known book [6]. The fact that the varieties $\mathbf{S l w} \mathbf{A}_{\mathbf{p}}$ are essentially infinitely based was proved in [12. The fact that there is no finite basis of identities for the semidirect product $\mathbf{S l} * \mathbf{A}_{\mathbf{p}}$ in the case $p=2$ was proved earlier, in [27. A basis of identities and a finite semigroup generating the variety $\mathbf{S l w R}_{\mathbf{1}}$ was found in [10. Bases of identities for the semidirect products of pseudovarieties $\mathbf{R}_{\mathbf{1}} * \mathbf{R}_{\mathbf{1}}$, $\mathbf{S l} * \mathbf{R}_{1}$, and $\mathbf{S l} * \mathbf{S l}$ can be found in the literature (see, for example, 20, 21, 30]). Furthermore, in 22] it was proved that there is no finite basis for the powers of the semidirect product $\mathbf{S l}^{\mathbf{n}}$ for any positive integer exponent $n \geq 3$. In addition, it has long been known that the atoms $\mathbf{N}_{\mathbf{2}}$ and $\mathbf{L}_{\mathbf{1}}$ are idempotents 28 . The structure of the lattice in the case of two group atoms of different prime exponents follows from Higman's result [6, Ch. 5, Theorem 54.41]. 
In $\S 2$ we calculate bases of identities of the wreath products of atoms of semigroup varieties; in certain cases at the same time we produce finite semigroups that generate the wreath products. In $\S 3$ we describe the finite lattices of subvarieties of wreath products or establish their infiniteness (finiteness). Finally, in $\S 4$ we find finite semigroups generating the wreath product of atoms of varieties, if this was not done before. Our main result, Theorem 1.2, is a consequence of Theorems 2.1,3.1, and 4.1 proved in $\S \S 2,3$, and 4 .

Theorems 2.1 and 4.1 were announced in 34. Theorem 3.1 substantially refines Theorem 3 in 34. All these results were published without proof in 13. We point out that in Theorem 3 in [13, due to an oversight, the cardinalities of the lattices $\mathcal{L}\left(\mathbf{R}_{\mathbf{1}} \mathbf{w} \mathbf{L}_{\mathbf{1}}\right)$, $\mathcal{L}\left(\mathbf{S l w} \mathbf{L}_{\mathbf{1}}\right), \mathcal{L}\left(\mathbf{R}_{\mathbf{1}} \mathbf{w} \mathbf{S l}\right)$, and $\mathcal{L}\left(\mathbf{A}_{\mathbf{p}} \mathbf{w} \mathbf{N}_{\mathbf{2}}\right)$ were indicated incorrectly, and a mistake was made in indicating the cardinality of the lattice $\mathcal{L}\left(\mathbf{S l w} \mathbf{N}_{\mathbf{2}}\right)$. The lower estimate for a possible cardinality of the latter lattice was made more precise in [14.

The author is grateful to the referee for some comments, which allowed us to improve our exposition and reduce the length considerably. In particular, the referee suggested that we introduce the definition of a Cross variety and state the main result as Theorem 1.2. In addition, the proofs of Propositions 2.31, 3.6, 3.12, and 3.17 were reduced.

\section{Calculation of Bases of identities OF WREATH PRODUCTS OF SEMIGROUP VARIETIES}

In this section we prove the following.

Theorem 2.1. The monoid wreath product $\mathbf{U w} \mathbf{V}$ of atoms of the lattice $\mathcal{L}$ of all semigroup varieties does not have a finite basis of identities if and only if $\mathbf{U}=\mathbf{S l}$ and $\mathbf{V}=\mathbf{A}_{\mathbf{p}}$ for some prime $p$. In this case the monoid wreath product of the atoms is essentially infinitely based.

In the proof, as a rule, we calculate the finite basis.

Remark 2.2. According to Proposition 3.3 in [10], the monoid wreath product $\mathbf{U w V}$ of two semigroup varieties is generated as a variety by all the extended standard wreath products of semigroups $S w R$, where $S \in \mathbf{U}$ and $R \in \mathbf{V}$. Consequently, to verify that an identity is true in the wreath product of varieties $\mathbf{U w} \mathbf{V}$, it is sufficient to verify that it is true in any extended standard wreath product of semigroups $S w R$, where $S \in \mathbf{U}$ and $R \in \mathbf{V}$. Furthermore, according to Algorithm 4.1 and Theorem 4.2 in [10, an identity

$$
u \equiv x_{i_{1}} \ldots x_{i_{k}}=y_{j_{1}} \ldots y_{j_{l}} \equiv v
$$

is true in the extended standard wreath product $S w R$ if and only if it is true in $R$ and the equality

$$
f_{1}(1) f_{2}\left(p_{1}\right) \ldots f_{k}\left(p_{1} \ldots p_{k-1}\right)=g_{1}(1) g_{2}\left(q_{1}\right) \ldots g_{l}\left(q_{1} \ldots q_{l-1}\right)
$$

is true in $S$ for any functions $f_{1}, \ldots, f_{k}, g_{1}, \ldots, g_{l} \in S^{A}$ and any values $p_{1}, \ldots, p_{k}$, $q_{1}, \ldots, q_{l} \in R$, where $A=R^{1}, S \in \mathbf{U}, R \in \mathbf{V}$. If equality (2.2) holds, then the semigroup $S$ satisfies the identity obtained from $(2.2)$ by replacing each factor $f_{i}\left(p_{1} \ldots p_{i-1}\right)$ or $g_{j}\left(q_{1} \ldots q_{j-1}\right)$ by a letter of the alphabet $X$ so that the following condition holds:

(*) Two factors are replaced by the same letter in $X$ if and only if their function symbols are the same and the equality of arguments is an identity that is true on the right $R$-polygon $A$.

In what follows we use the following notation: $I(S)$ is the set of identities that are true in a semigroup $S, I(\mathbf{V})$ is the set of identities that are true in a variety $\mathbf{V}, \operatorname{var}\left\{u_{1}=v_{1}\right.$, $\left.\ldots, u_{k}=v_{k}\right\}$ is the variety of all semigroups in which the identities $u_{1}=v_{1}, \ldots, u_{k}=v_{k}$ are true, var $S$ is the semigroup variety generated by a semigroup $S$. 
Lemma 2.3. If $S$ is a non-one-element right-singular semigroup, then

$$
I(S w R)=\{u z=v z: u=v \in I(R)\} .
$$

Proof. By Remark 2.2 it is sufficient to check under what conditions equality (2.2) always holds in $S$. According to Remark 2.2, the identity (2.1) is true in the wreath product $S w R$ if and only if it is true in $R$ and the identity obtained from equality (2.2) by using the rule $(*)$ is satisfied by $S$. It is easy to verify that equality $(2.2)$ holds identically in $S$ if and only if the identity has the form $u z=v z$, where $u=v \in I(R)$.

Corollary 2.4. The set of identities $I\left(\mathbf{R}_{\mathbf{1}} \mathbf{w} \mathbf{V}\right)=\{u z=v z: u=v \in I(\mathbf{V})\}$.

Corollary 2.5. The following equalities are valid:

a) $\mathbf{R}_{\mathbf{1}} \mathbf{w} \mathbf{N}_{\mathbf{2}}=\operatorname{var}\left\{x_{1} x_{2} z=y_{1} y_{2} z\right\}$

b) $\mathbf{R}_{\mathbf{1}} \mathbf{w} \mathbf{L}_{\mathbf{1}}=\mathbf{R}_{\mathbf{1}} \vee \mathbf{L}_{\mathbf{1}} \vee \mathbf{N}_{\mathbf{2}}=\operatorname{var}\{x y z=x z\}$;

c) $\mathbf{R}_{1}^{2}=\operatorname{var}\left\{x z_{1} z_{2}=z_{1} z_{2}\right\}$

d) $\mathbf{R}_{\mathbf{1}} \mathbf{w S l}=\operatorname{var}\left\{x z=x^{2} z, x y z=y x z\right\}$;

e) $\mathbf{R}_{\mathbf{1}} \mathbf{w} \mathbf{A}_{\mathbf{n}}=\mathbf{R}_{\mathbf{1}} \vee \mathbf{A}_{\mathbf{n}}=\operatorname{var}\left\{z=x^{n} z, x y z=y x z\right\}$.

The following lemma is merely a re-formulation of Lemma 4.4 in [15].

Lemma 2.6 (see Lemma 4.4 in [15]). Let $\mathbf{U}$ be a semigroup variety and let

$$
\begin{aligned}
I_{1} & =\{x u=x v: u=v \in I(\mathbf{U}), x \in X\}, \\
I & =\{x u=x v: z u=z v \in I(\mathbf{U}) \text { for any } z \in X\}, \\
I_{2} & =\{x u=x v: z u=z v \in I(\mathbf{U}) \text { for some } z \in X\} .
\end{aligned}
$$

Then the set $I\left(\mathbf{U w}_{\mathbf{1}}\right)$ of identities that are true in the monoid wreath product of the semigroup variety $\mathbf{U}$ and the variety $\mathbf{L}_{\mathbf{1}}$ of semigroups of left zeros coincides with the set $I$, and the following inclusions hold:

$$
I_{1} \subseteq I \subseteq I_{2} .
$$

The following assertion follows immediately from Lemma 4.5 in [15].

Lemma 2.7. If $B$ is a basis of identities of a periodic variety $\mathbf{U}$, and an identity of the form

$$
z=z x^{m}
$$

is true in $\mathbf{U}$, then the set of identities

$$
B_{1}=\left\{z w=z w^{\prime}: w=w^{\prime} \in B, z \notin c\left(w w^{\prime}\right)\right\}
$$

is a basis of identities of the wreath product $\mathbf{U w} \mathbf{L}_{\mathbf{1}}$ of the semigroup varieties $\mathbf{U}$ and $\mathbf{L}_{\mathbf{1}}$.

Corollary 2.8. The wreath product $\mathbf{A}_{\mathbf{m}} \mathbf{w} \mathbf{L}_{\mathbf{1}}=\operatorname{var}\left\{x y z=x z y, x=x y^{m}\right\}$.

Lemma 2.9. The set of identities $I\left(\mathbf{S l w} \mathbf{L}_{\mathbf{1}}\right)$ of the wreath product of varieties coincides with the set

$$
\begin{aligned}
I_{0}=\{u=v: \text { 1) } c(u)= & \left.c(v), 2) h_{1}(u)=h_{1}(v), 3\right) \text { if } z=h_{1}(u), \\
& \text { then either } \left.|u|_{z}=|v|_{z}=1 \text { or }|u|_{z} \geq 2,|v|_{z} \geq 2\right\} .
\end{aligned}
$$

Proof. Applying Lemma 2.5 we obtain that the equalities

$$
I(\mathbf{W})=\{x u=x v: z u=z v \in I(\mathbf{S l}) \text { for any } z \in X\}=I
$$

and the inclusion

$$
I \supseteq\{z u=z v: u=v \in I(\mathbf{S l})\}=I_{1}
$$

hold for the wreath product $\mathbf{W}=\mathbf{S l w}_{\mathbf{1}}$. It is easy to see that the set of identities $I(\mathbf{W})$ coincides with the set $I_{0}$. 
Lemma 2.10. The wreath product $\mathbf{S l w}_{\mathbf{1}}=\operatorname{var}\left\{x y z=x z y, x y=x y^{2}\right\}$.

Proof. We verify directly that the identities

$$
z x y=z y x, \quad z x=z x^{2}
$$

belong to the set $I(\mathbf{W})$. On the other hand, if $u=v \in I_{0}$, then $u \equiv z u^{\prime}=z v^{\prime} \equiv v$, where either $z \notin c\left(u^{\prime} v^{\prime}\right)$ or $c\left(u^{\prime}\right)=c\left(v^{\prime}\right)$ involves the letter $z$. The fact that $c\left(u^{\prime}\right)=c\left(v^{\prime}\right)$ implies that the identity $u^{\prime}=v^{\prime}$ can be derived from the identities $x y=y x$ and $x=x^{2}$. Consequently, the identity $u=v$ can be derived from (2.8).

Lemma 2.11. If $\operatorname{var} S \supseteq \mathbf{S l}$, then an identity $u=v$ belongs to $I(S w R)$, where $R \in \mathbf{N}_{\mathbf{2}}$, if and only if the following conditions hold: 1) either $u=v$ is a trivial identity or $|u|,|v| \geq 3$; 2) $h_{2}(u)=h_{2}(v)$; 3) replacing the common beginning $h_{2}(u)=h_{2}(v)$ in the identity $u \equiv h_{2}(u) u_{1}=h_{2}(v) v_{1} \equiv v$ by the subword $z_{1} z_{2}$ results in the identity $z_{1} z_{2} u_{1}=$ $z_{1} z_{2} v_{1}$, where $c\left(u_{1}\right)=c\left(v_{1}\right)$ and $z_{1}, z_{2} \notin c\left(u_{1}\right)$, which is true in the variety $\operatorname{var} S$.

Proof. Obviously, $\operatorname{var}(S w R) \supseteq \mathbf{N}_{\mathbf{2}}$, and therefore $|u|,|v| \geq 2$ for a non-trivial identity. By Remark 2.2, the inclusion $u=v \in I(S w R)$ means that $|u|,|v| \geq 2$, and for any $p, q \in R$ the equality

$$
f_{1}(1) f_{2}(p) f_{3}(0) \ldots f_{k}(0)=g_{1}(1) g_{2}(q) g_{3}(0) \ldots g_{k}(0)
$$

is true in the semigroup $S$. Choosing for the semigroup $S$ one of its divisors, given by the two-element semilattice $U_{2}=\{0,1\}$ we can argue as follows. If $f_{1} \neq g_{1}$ in (2.9), then we set $f_{1}(1)=0$ and $f_{1}(a)=1$ for $a \in R$, while the other functions occurring in (2.9), including $g_{1}$, are set to be equal to the function $c_{1}$ that is identically equal to 1 . Obviously, then the left-hand side of equality (2.9) takes the value 0 , while the right-hand side takes the value 1 . Similarly, if $f_{2} \neq g_{2}$, then we choose for $p$ a non-zero element in $R$ and set $f_{2}(p)=0$ and $f_{2}(a)=1$ for any $a \in R \backslash\{p\}$, while the other functions occurring in (2.9), including $g_{2}$, are set to be equal to the function $c_{1}$. Then (2.9) again gives a false equality. Consequently, $h_{2}(u)=h_{2}(v)$. Moreover, according to Remark 2.2, from equality (2.9) one can deduce that $S$ satisfies the identity $u \equiv z_{1} z_{2} u_{1}=z_{1} z_{2} v_{1} \equiv v$, where $z_{1}, z_{2} \notin c\left(u_{1}\right) \cup c\left(v_{1}\right)$. Furthermore, by the equality $c(u)=c(v)$ for an identity that is true in $S$, since var $S \supseteq \mathbf{S l}$, this implies condition 3) of the lemma and, in particular, $c\left(u_{1}\right)=c\left(v_{1}\right)$. Furthermore, condition 2) for a non-trivial identity $u=v$ implies the validity of condition 1$)$. The lemma is proved.

The next corollary follows easily from the description of the identities of the wreath product of semigroups obtained in Lemma 2.11.

Corollary 2.12. If $\mathbf{U} \supseteq \mathbf{S l}$, then $\mathbf{U w N}_{2} \supseteq \mathbf{U} \vee \mathbf{N}_{2} \vee \mathbf{L}_{1}$.

Corollary 2.13. If $\operatorname{var} S=\mathbf{S l}$, then an identity $u=v$ belongs to $I(S w R)$, where $R \in \mathbf{N}_{\mathbf{2}}$ and $|R| \geq 2$, if and only if the following conditions hold: 1) either $u=v$ is a trivial identity or $|u|,|v| \geq 3$; 2) $h_{2}(u)=h_{2}(v)$; 3) replacing the common beginning $h_{2}(u)=h_{2}(v)$ in the identity $u \equiv h_{2}(u) u_{1}=h_{2}(v) v_{1} \equiv v$ by the subword $z_{1} z_{2}$, where $z_{1}, z_{2} \notin c(u v)$, yields the identity $z_{1} z_{2} u_{1}=z_{1} z_{2} v_{1}$ where $c\left(u_{1}\right)=c\left(v_{1}\right)$.

Corollary 2.14. The wreath product $\mathbf{S l w N}_{2}$ is equal to $\operatorname{var}\left\{x_{1} x_{2} y z=x_{1} x_{2} z y\right.$, $\left.x_{1} x_{2} y=x_{1} x_{2} y^{2}\right\}$.

Lemma 2.15. The wreath product $\mathbf{A}_{\mathbf{m}} \mathbf{w} \mathbf{N}_{\mathbf{2}}$ is equal to $\operatorname{var}\left\{x_{1} x_{2} y z=x_{1} x_{2} z y\right.$, $\left.x_{1} x_{2}=x_{1} x_{2} y^{m}\right\}$.

Proof. Starting the calculations as in Lemma 2.11 we observe that, according to Remark 2.2, equality (2.9) means that the identity

$$
y_{1} y_{2} u^{\prime}=z_{1} z_{2} v^{\prime}
$$


such that $y_{1}, y_{2}, z_{1}, z_{2} \notin c\left(u^{\prime} v^{\prime}\right)$ is true in the semigroup $S$. Setting $y_{1}=y_{2}=z_{1}=z_{2}=1$ in (2.10) we now obtain that the identity $u^{\prime}=v^{\prime}$ is true in the semigroup $S=C(m)$, where $C(m)$ is the cyclic group of order $m$. Clearly, all such identities follow as a consequence of the group basis $\left\{x y=y x, y^{m}=1\right\}$. Consequently, all the identities of the wreath product of varieties $\mathbf{A}_{\mathbf{m}} \mathbf{w} \mathbf{N}_{\mathbf{2}}$ are a consequence of the basis of identities

$$
\left\{x_{1} x_{2} y z=x_{1} x_{2} z y, x_{1} x_{2}=x_{1} x_{2} y^{m}\right\},
$$

and the lemma is proved.

Lemma 2.16. An identity $u=v$ is true in SlwSl if and only if $c(u)=c(v)$ and, moreover, for any occurrence of a variable $y$ in $u \equiv u_{1} y u_{2}$ there exists an occurrence of $y$ in $v \equiv v_{1} y v_{2}$ such that $c\left(u_{1}\right)=c\left(v_{1}\right)$ and, conversely, for any occurrence of a variable $y$ in $v \equiv v_{1} y v_{2}$ there exists an occurrence of $y$ in $u \equiv u_{1} y u_{2}$ such that $c\left(u_{1}\right)=c\left(v_{1}\right)$.

Proof. Indeed, the inclusion SlwSl $\supseteq \mathbf{S l}$ is equivalent to the equality $c(u)=c(v)$, and equality (2.2) induces an identity on the passive semigroup of the wreath product, which holds if and only if for any integer $i=1,2, \ldots, k$ there exists an integer $j=1,2, \ldots, l$ such that

$$
f_{i}\left(p_{1} \ldots p_{i-1}\right)=g_{j}\left(q_{1} \ldots q_{j-1}\right),
$$

and, conversely, for any integer $j=1,2, \ldots, l$ there exists an integer $i=1,2, \ldots, k$ such that equality $(2.12)$ holds. The latter condition means precisely that for any integer $i=1,2, \ldots, k$ there exists an integer $j=1,2, \ldots, l$ such that $f_{i}=g_{j}$ and $p_{1} \ldots p_{i-1}=$ $q_{1} \ldots q_{j-1}$, and conversely. Furthermore, the first of these equalities means that the symbols $f_{i}$ and $g_{j}$ are substituted for one and the same variable $y$ of the identity $u=v$, and the fact that the second equality is true in $\mathbf{S l}$ means that $c\left(u_{1}\right)=c\left(v_{1}\right)$ for these occurrences of $y$ in $u$ and in $v$. The lemma is proved.

Proposition 2.17. The set of identities

$$
\begin{aligned}
x^{2} & =x^{3}, \quad x y x^{2}=x y x \\
\text { xuyvy } x=\text { xuyvxy }, \quad \text { xyyx } & =x y x y, \quad \text { xuyy } x=x u y x y, \quad \text { xyvy } x=x y v x y
\end{aligned}
$$

is a basis of identities of the wreath product of varieties SlwSl.

Proof. It is easy to verify that the identities (2.13) satisfy the conditions of Lemma 2.16 and therefore they are true in SlwSl. Next, every word $u$ can be written in the form

$$
u \equiv x_{1} u_{1} x_{2} u_{2} \ldots x_{n} u_{n},
$$

where we have distinguished the first occurrences of the variables $x_{1}, x_{2}, \ldots, x_{n}$ on which the word $u$ depends, and the word $u_{i}$ depends only on the variables $x_{1}, x_{2}, \ldots, x_{i}$, and $u_{i}$ can also be the empty word. Here we call a word of the form (2.14) a reduced word if the $u_{i}$ are linear words, and the variables in them are ordered according to increasing indices. It is easy to see that by using identity (2.13) one can reduce any word to a reduced word that is equal to it in SlwSl. In order to show that the set of identities (2.13) is a basis of identities for SlwSl it is now sufficient to show that any two different reduced words are not equal in SlwSl. To do this we will show that any identity $u=v$ in SlwSl satisfies the following three conditions:

1) $c(u)=c(v)$;

2) the order of occurrence of the variables in the words $u$ and $v$ is the same;

3 ) for reduced words $u$ and $v$ we have $u_{i}=v_{i}$ for any $i=1,2, \ldots, n$.

Condition 1) is obvious. If condition 2) is violated, then the condition of Lemma 2.16 does not hold. Indeed, suppose that

$$
u \equiv x_{1} u_{1} \ldots x_{i-1} u_{i-1} x_{i} u^{\prime}=x_{1} v_{1} \ldots x_{i-1} v_{i-1} y_{i} v^{\prime} \equiv v
$$


for some $i$ and $x_{i} \neq y_{i}$. We set $x_{i}=\left(c_{1}, 0\right)$ and $y_{i}=(g, 0)$, where $g(1)=0$ and $g(0)=1$, while the other variables are set to be equal to $\left(c_{1}, 1\right)$. Then equality $(2.2)$ takes the form

$$
f_{1}(1) \ldots f_{i}(1) f_{r}(0) \ldots=g_{1}(1) \ldots g_{i}(1) g_{s}(0) \ldots
$$

We observe that the right-hand side of equality (2.16) is equal to zero, since it contains the factor $g(1)=0$, while the left-hand side of equality (2.16) is equal to 1 , since it does not contain the factor $g(1)$. Now suppose that in the identity $u=v$ the words are reduced but condition 3 ) is violated. Then for some $i \geq 1$ equality (2.15) holds in which either both words $u^{\prime}, v^{\prime}$ are empty or both begin with one and the same variable $x_{i+1}$. Suppose that $u_{i} \neq v_{i}$. For reduced words $u$ and $v$, this means that some variable $x_{l}$ $(l=1,2, \ldots, i)$ occurs in exactly one of the words $u_{i}$ or $v_{i}$. Suppose for definiteness that $x_{l} \in c\left(u_{i}\right) \backslash c\left(v_{i}\right)$. Suppose that the semigroup $R$ contains a subsemigroup isomorphic to the three-element chain $U_{3}=\{1, d, 0\}$. We use the homomorphism $\varphi\left(x_{j}\right)=\left(f_{j}, p_{j}\right)$ $(j=1,2, \ldots, n)$ and in equality $(2.15)$ we set $p_{1}=\cdots=p_{i-1}=1, p_{i}=d, p_{i+1}=0$, $f_{l}=g$, where $g(1)=g(0)=1$ and $g(d)=0$, while the other functions in (2.15) are set to be equal to the function $c_{1}$ that is identically equal to 1 . Then on the left-hand side of equality (2.15) the first projection contains the factor $g(d)=0$ and, consequently, it is equal to 0 , while on the right-hand side it does not contain such a factor and therefore it is equal to 1 . The contradiction thus obtained shows that condition 3) is satisfied. The proposition is proved.

Analyzing the proof of Proposition 2.17 one can see that for the calculation of a basis of identities for the wreath product of semigroups $S w R$, where $S, R \in \mathbf{S l}$, the assumptions were used that the semigroup $S$ is non-one-element and therefore the twoelement semilattice $U_{2}$ is a divisor of it, while the semigroup $R$ contains a three-element chain $U_{3}$ as a subsemigroup. This observation allows us to state the following result.

Corollary 2.18. The wreath product $\mathbf{S l w S l}=\operatorname{var}\left(U_{2} w U_{3}\right)$, where $U_{2}$ is the two-element chain and $U_{3}$ is the three-element chain.

Remark 2.19. The identities

$$
\begin{aligned}
x^{2} y x & =x y x, \\
r x y x z x & =x y z x
\end{aligned}
$$

are true in the wreath product $U_{2} w U_{2}$, but they are false in $U_{2} w U_{3}$. Consequently, SlwSl $\neq \operatorname{var}\left(U_{2} w U_{2}\right)$.

Proof. Indeed, it is easy to see that both identities (2.17) and (2.18) do not satisfy the conditions of Lemma 2.16. Therefore they do not hold in SlwSl and, consequently, by Corollary 2.18, also in the wreath product $\operatorname{var}\left(U_{2} w U_{3}\right)$. On the other hand, using the homomorphism $\varphi$ such that $\varphi(x)=(f, p), \varphi(y)=(g, q), \varphi(z)=(h, r)$ we obtain from the identity (2.17) the equality

$$
f(1) f(p) g(p) f(p q)=f(1) g(p) f(p q) .
$$

When $p=1$ equality (2.19) induces the identity $x^{2} y z=x y z$ in $S=U_{2}$, and when $p=0$ the identity $x y z y=x z y$. Both identities are true in Sl. Therefore by Theorem 4.2 in [10] (see Remark 2.1) the identity (2.17) is true in the wreath product $\operatorname{var}\left(U_{2} w U_{2}\right)$. The fact that the identity $(2.18)$ is true in the wreath product $\operatorname{var}\left(U_{2} w U_{2}\right)$ can be verified in similar fashion. The remark is proved.

Lemma 2.20. An identity $u=v$ is true in $\mathbf{A}_{\mathbf{m}} \mathbf{w S l}$ if and only if 1) $c(u)=c(v)$, 2) $h_{1}(u)=h_{1}(v)$, and, also 3) for any variable $y$ the difference between the number of occurrences of $y$ in $u \equiv u_{1} y u_{2}$ and the number of occurrences of $y$ in $v \equiv v_{1} y v_{2}$ when $c\left(u_{1}\right)=c\left(v_{1}\right)$ is a multiple of $m$. 
Proof. According to Remark 2.2, the identity (2.1) is true in $\mathbf{A}_{\mathbf{m}} \mathbf{w} \mathbf{S l}$ if and only if $c(u)=c(v)$ and, in addition, equality $(2.2)$ is true for any $p_{1}, \ldots, p_{k}, q_{1}, \ldots, q_{l} \in R$ and any functions $f_{1}, \ldots, f_{k}, g_{1}, \ldots, g_{l} \in F\left(R^{1}, S\right)$, where $R \in \mathbf{S l}$ and $S \in \mathbf{A}_{\mathbf{m}}$. Hence in (2.2) we have $f_{1}=g_{1}$, that is, $h_{1}(u)=h_{1}(v)$. Furthermore, the rule $(*)$ given in Remark 2.2 implies that in equality $(2.2)$ the numbers of occurrences of any factor $f_{i}\left(p_{1} \ldots p_{i-1}\right)$ on the left- and right-hand side can differ only by a number that is a multiple of $m$.

Corollary 2.21. Every non-trivial permutational identity is false in $C(m) w R$, where $C(m)$ is the cyclic group of order $m$ and $R \in \mathrm{Sl}$.

Lemma 2.22. The identities

$$
\begin{aligned}
x & =x^{1+m}, \\
x y^{2} x & =x y x y
\end{aligned}
$$

are true in the wreath product of varieties $\mathbf{A}_{\mathbf{m}} \mathbf{w} \mathbf{S l}$.

Proof. This fact follows immediately from Lemma 2.20 , or it can be verified by a straightforward calculation in the wreath product of semigroups $S w R$, where $R \in \mathbf{S l}$ and $S \in \mathbf{A}_{\mathbf{m}}$.

Lemma 2.23. If the identity (2.20) is true in a semigroup variety, then the identity (2.21) is true in it if and only if the identity

$$
\text { xyvy } x=\text { xyvxy }
$$

is true in it.

Proof. If (2.20) and (2.22) are true in a variety, then the identity (2.21) is also true in it:

$$
x y y x=x y y^{m} y x=x y y^{m} x y=x y x y .
$$

Conversely, if (2.20) and (2.21) are true, then (2.22) is true:

$$
\begin{aligned}
\text { xyvyx } & =(x y v)^{m-1} \text { xyvxyvyx }=(x y v)^{m-1} x y v^{2} x y y x \\
& =(x y v)^{m-1} x y v^{2} x y x y=(x y v)^{m-1} \text { xyvxyvxy }=x y v x y .
\end{aligned}
$$

The lemma is proved.

Lemma 2.24. If the identities (2.20) and (2.21) are true in a semigroup variety, then the identities (2.22) are also true in it and

$$
\begin{aligned}
x y x^{m} & =x y, \\
\text { xuyvyx } & =\text { xuyvxy, } \\
\text { xuyyx } & =\text { xuyxy. }
\end{aligned}
$$

Proof. The fact that (2.22) is true follows from Lemma 2.23. Next, using identities (2.20) and (2.22) we obtain

$$
x y=x y(x y)^{m}=x y y^{m}(x y)^{m}=x y y^{m} x^{m} y^{m}=x y y^{2 m} x^{m}=x y x^{m} .
$$

Consequently, the identity (2.23) is true in the variety. We now prove that (2.24) is true. Indeed, using (2.20) and (2.22) we obtain

$$
\text { xuyvy } x=(\text { xuy })^{m-1} \text { xuyx }(\text { uyv }) y x=(x u y)^{m-1} \text { xuyxuyvxy }=\text { xuyvxy. }
$$

Finally, it is easy to see that (2.20) and (2.24) imply (2.25):

$$
\text { xuyy } x=\text { uyy }^{m} y x=x u y y^{m} x y=x u y x y .
$$

Proposition 2.25. The set of two identities (2.20) and (2.21) is a basis of identities of the wreath product of varieties $\mathbf{A}_{\mathbf{m}} \mathbf{w S l}$. 
Proof. According to Lemmas 2.22 and 2.24, the identities (2.22), (2.23), (2.24), and (2.25) are also true in the variety $\mathbf{A}_{\mathbf{m}} \mathbf{w S l}$. It is easy to see that by using the identities (2.20)-(2.25) one can reduce any word to the form (2.14) in which we pick out the first occurrences of the variables $x_{1}, \ldots, x_{n}$ in the word $u$, while the words $u_{i}$ depend only on the variables $x_{1}, \ldots, x_{i}$, and can also be empty words. We say that a word of the form (2.14) is reduced if $u_{i} \equiv x_{1}^{a_{1}} \ldots x_{i}^{a_{i}}$, where $0 \leq a_{j} \leq m-1(j=1, \ldots, i)$. We claim that any two different reduced words are not equal in the variety $\mathbf{A}_{\mathbf{m}} \mathbf{w S}$. For that we will show that for any identity $u=v$ of the variety $\mathbf{A}_{\mathbf{m}} \mathbf{w S l}$ the following conditions hold: 1) $c(u)=c(v)$; 2) the order in which the variables occur in the words $u$ and $v$ is the same; 3) for reduced words $u$ and $v$ we have $u_{i} \equiv v_{i}$ for any $i=1,2, \ldots, n$. Condition 1) obviously holds, since $\mathbf{A}_{\mathbf{m}} \mathbf{w} \mathbf{S l} \supseteq \mathbf{S l}$. If condition 2) is violated, then the conditions of Lemma 2.20 do not hold. Indeed, suppose that condition 2) is violated. Then, for the identity $u=v$, equality (2.15) holds for some $i$ and $x_{i} \neq y_{i}$. Assuming that the semigroup $S \in \mathbf{A}_{\mathbf{m}}$ is non-one-element and, consequently, contains an element $b \neq 1$, while the semilattice $R \in \mathbf{S}$ contains the two-element chain $U_{2}=\{d, 1\}$, in the identity $(2.15)$ we set $\varphi\left(x_{i}\right)=\left(c_{1}, d\right)$ and $\varphi\left(y_{i}\right)=(g, d)$, where $g(1)=b \neq 1$ and $g(a)=1$ for $a \in R \backslash\{1\}$, while $\varphi\left(x_{j}\right)=\left(c_{1}, 1\right)$ for the other variables, that is, for $x_{j} \notin\left\{x_{i}, y_{i}\right\}$. Then the first projection of the left-hand side of equality (2.15) is a function $F_{1}(a)$ that takes the value 1 at the point $a=1$, while the first projection of the right-hand side of equality (2.15) is a function $F_{2}(a)$ that takes the value $b$ at the point $a=1$, since it contains the factor $g(1)=b$ exactly once.

Now suppose that the identity $u=v$ satisfies conditions 1 ) and 2), while condition 3) is violated. Then this identity induces the equality

$$
u \equiv x_{1} u_{1} \ldots u_{i-1} x_{i} u_{i} u^{\prime}=x_{1} v_{1} \ldots x_{i-1} v_{i-1} x_{i} v_{i} v^{\prime} \equiv v
$$

in which either both the words $u^{\prime}, v^{\prime}$ are empty or both of them begin with exactly the same variable $x_{i+1}$. Suppose that $u_{i} \not \equiv v_{i}$. For reduced representations we have $u_{i} \equiv x_{1}^{k_{1}} \ldots x_{i}^{k_{i}}$ and $v_{i} \equiv x_{1}^{l_{1}} \ldots x_{i}^{l_{i}}$. Suppose that $k_{j} \neq l_{j}$ for some $1 \leq j \leq i$. Suppose that the semigroup $R$ contains the three-element chain $U_{3}=\{1, d, 0\}$. We consider the homomorphism $\varphi_{i}\left(x_{j}\right)=\left(f_{j}, p_{j}\right)(j=1, \ldots, n)$ and set $p_{1}=\cdots=p_{i-1}=1, \quad p_{i}=d$, $p_{i+1}=0, \quad f_{i}=g$, where $g(d)=b$ and $g(a)=1$ for $a \in R \backslash\{d\}$, while all the other functions in (2.26) are set to be equal to the function $c_{1}$ that is identically equal to 1 . Then the identity (2.26) induces an equality of the first projections of elements of the wreath product $S w R$ with the left-hand side equal to $g(d)^{k_{i}}=b^{k_{i}}$, while the right-hand side is equal to $g(d)^{l_{i}}=b^{l_{i}}$. Hence, $k_{j}-l_{j} \equiv 0(\bmod m)$. For reduced representations of the words $u$ and $v$, this implies that $k_{j}=l_{j}$ for any $1 \leq j \leq i$. The lemma is proved.

Corollary 2.26. The wreath product $\mathbf{A}_{\mathbf{m}} \mathbf{w} \mathbf{S l}=\operatorname{var}\left(C(m) w U_{2}\right)$, where $U_{2}$ is the twoelement chain.

Proof. To prove the assertion, first of all we observe that in the proof of Proposition 2.25, the fact that the active semigroup $R$ of the wreath product of semigroups contains a subsemigroup isomorphic to the three-element chain was used only in the very last part of the proof. We now modify this last argument. Namely, we consider the homomorphism $\psi_{i}$ of a free semigroup into the wreath product $S w R$ that differs from the homomorphism $\varphi_{i}$ indicated above by the fact that in it we choose the function $g: U_{2} \rightarrow C(m)$ so that $g(1)=1$ and $g(0)=b \neq 1$. Then the identity (2.26) induces an equality such that the first projection of the left-hand side equals $g(0)^{k_{i}+\cdots+k_{n}}=b^{k_{i}+\cdots+k_{n}}$, while the first projection of the right-hand side is equal to $g(0)^{l_{i}+\cdots+l_{n}}=b^{l_{i}+\cdots+l_{n}}$. Based on the equality of these sides in the group, we obtain that

$$
\left(l_{i}+\cdots+l_{n}\right)-\left(k_{i}+\cdots+k_{n}\right) \equiv 0(\bmod m) .
$$


Next, setting $i=n$ in equality (2.27) we obtain $k_{n}=l_{n}$, setting $i=n-1$ we obtain $k_{n-1}=l_{n-1}$, and so on. Thus, we find that two reduced representations for equal words in the wreath product of varieties $\mathbf{A}_{\mathbf{m}} \mathbf{w} \mathbf{S l}$ coincide.

To obtain a basis of identities for the variety $\mathbf{A}_{\mathbf{m}} \mathbf{w} \mathbf{R}_{\mathbf{1}}$ we prove several preliminary assertions. In this case we do not follow the usual method that relies on the description of all the identities of the wreath product of varieties; instead, we use information about the structure of the semigroups of the wreath product of varieties.

Lemma 2.27. The identity

$$
x=(x y)^{m} x
$$

is true in the wreath product of varieties $\mathbf{A}_{\mathbf{m}} \mathbf{w} \mathbf{R}_{\mathbf{1}}$. Consequently, the variety $\mathbf{A}_{\mathbf{m}} \mathbf{w} \mathbf{R}_{\mathbf{1}}$ is a completely simple variety over a group of exponent $m$.

Proof. For the wreath product $S w R$ we set $\varphi(x)=(f, p), \varphi(y)=(g, q)$. Then

$$
\begin{aligned}
((f, p)(g, q))^{m}(f, p) & =\left(f\left({ }^{p} g\right), p q\right)^{m}(f, p) \\
& =\left(f\left({ }^{p} g\right)\left({ }^{p q} f\right)\left({ }^{p q p} g\right) \ldots\left({ }^{(p q)^{m-1}} p\right),(p q)^{m}\right)(f, p) \\
& =\left(f\left({ }^{p} g^{q} f\right)^{m-1}\left({ }^{p} g\right), q\right)(f, p)=\left(f\left({ }^{p} g^{q} f\right)^{m}, p\right)=(f, p) .
\end{aligned}
$$

Thus, identity (2.28) is true in the wreath product of varieties $\mathbf{A}_{\mathbf{m}} \mathbf{w} \mathbf{R}_{\mathbf{1}}$. Next, as already mentioned in the proof of Proposition 4.2 in [12], the fact that the identity (2.28) is true in a variety means that all semigroups of this variety are completely simple semigroups over a group of exponent $m$. This implies the second assertion of the lemma.

Lemma 2.28. The inclusion $\mathbf{A}_{\mathbf{m}} \mathbf{w} \mathbf{R}_{\mathbf{1}} \supseteq \mathbf{A}_{\mathbf{m}} \vee \mathbf{R}_{\mathbf{1}} \vee \mathbf{L}_{\mathbf{1}}$ holds.

Proof. By Proposition 3.4 in [10, we have $\mathbf{A}_{\mathbf{m}} \mathbf{w} \mathbf{R}_{\mathbf{1}} \supseteq \mathbf{A}_{\mathbf{m}} \vee \mathbf{R}_{\mathbf{1}}$. Now we apply the algorithm described in Remark 2.2 to an arbitrary identity (2.1) that is true in the wreath product $\mathbf{A}_{\mathbf{m}} \mathbf{w} \mathbf{R}_{\mathbf{1}}$. Then we find that in equality (2.2), both on the left- and right-hand side, the argument 1 occurs only for the first functions-factors. Therefore, according to the rule $(*)$ of Remark (2.2), either $h_{1}(u)=h_{1}(v)$, or the semigroup $S \in \mathbf{A}_{\mathbf{m}}$ must satisfy an identity of the form $y u_{1}=z v_{1}$, where $y \neq z$ and $y, z \notin c\left(u_{1} v_{1}\right)$. Since the latter is possible only in the one-element group, the first assumption is true, that is, $h_{1}(u)=h_{1}(v)$. Hence the inclusion $\mathbf{A}_{\mathbf{m}} \mathbf{w} \mathbf{R}_{\mathbf{1}} \supseteq \mathbf{L}_{\mathbf{1}}$ also holds. The lemma is proved.

The last two lemmas show us another path in the search for a basis of identities of the variety $\mathbf{A}_{\mathbf{m}} \mathbf{w} \mathbf{R}_{\mathbf{1}}$.

Definition 2.29. We define the semigroup $S_{m}$ as the Rees semigroup of matrix type $M(C(m), I, \Lambda, P)$, where $C(m)$ is the cyclic group of order $m$ with a generator $a$ and identity element $e, I=\Lambda=\{1,2\}$, and the matrix $P$ coincides with the matrix of order 2 over this group,

$$
\left(\begin{array}{ll}
e & e \\
e & a
\end{array}\right)
$$

By Rasin's theorem (see [16. Theorem 20.1] or [7, 31]), the semigroup $S_{m}$ has a basis of identities formed by three identities: namely, the identity (2.28) and the following two identities:

$$
\begin{aligned}
x^{2} y x & =x y x^{2}, \\
\left(x^{m} y^{m}\right)^{m} & =(x y)^{m} .
\end{aligned}
$$


Lemma 2.30. If the identities (2.28) and (2.29) are true in a variety $\mathbf{U}$, then the identities

$$
x y x^{i}=x^{i} y x
$$

for all positive integers $i \geq 2$ and the identity (2.30) are also true in $\mathbf{U}$.

Proof. The fact that the identity (2.31) is true can be easily verified by induction on $i$. Next, (2.28) obviously implies that the identity (2.20) is true in U. Using (2.31) and $(2.20)$ we obtain the chain of identities

$$
\begin{aligned}
\left(x^{m} y^{m}\right)^{m} & =x^{m} y^{m} x^{m} y^{m} \ldots x^{m} y^{m}=x y^{m} x^{2 m-1} y^{m} \ldots x^{m} y^{m} \\
& =x y^{m} x y^{m} \ldots x^{1+m(m-1)} y^{m}=x y^{m} x y^{m} \ldots x y^{m} \\
& =x y x y^{2 m-1} \ldots x y^{m}=x y x y \ldots x y^{1+m(m-1)}=(x y)^{m} .
\end{aligned}
$$

The lemma is proved.

Proposition 2.31. The wreath product of semigroup varieties $\mathbf{A}_{\mathbf{m}} \mathbf{w} \mathbf{R}_{\mathbf{1}}$ coincides with the variety generated by the semigroup $S_{m}$ and so it has a basis of identities consisting of the two identities (2.28) and (2.29).

Proof. According to Lemma 2.27, the identity (2.28) is true in $\mathbf{A}_{\mathbf{m}} \mathbf{w} \mathbf{R}_{\mathbf{1}}$. By Remark 2.2 it is sufficient to verify that the identity (2.29) is true in any wreath product of semigroups $S w R$, where $S \in \mathbf{A}_{\mathbf{m}}$ and $R \in \mathbf{R}_{\mathbf{1}}$. Using the homomorphism $\varphi(x)=(f, p), \varphi(y)=(g, q)$ we obtain

$$
(f, p)^{2}(g, q)(f, p)=\left(f\left({ }^{p} f\right)\left(p^{2} g\right)\left({ }^{2} q f\right), p^{2} q p\right)=\left(f\left({ }^{p} f\right)\left({ }^{p} g\right)\left({ }^{q} f\right), p\right),
$$

and, similarly,

$$
(f, p)(g, q)(f, p)^{2}=\left(f\left({ }^{p} g\right)\left({ }^{q} f\right)\left({ }^{p} f\right), p\right) .
$$

Since the semigroup $S$ is commutative, it follows that the identity (2.29) is true in the wreath product $S w R$. Consequently, $\mathbf{A}_{\mathbf{m}} \mathbf{w} \mathbf{R}_{\mathbf{1}} \subseteq \operatorname{var} S_{m}$.

We now show that $S_{m} \in \mathbf{A}_{\mathbf{m}} \mathbf{w} \mathbf{R}_{\mathbf{1}}$. To do this we observe that by virtue of the proof of Proposition XI.3.1 in the book [23], the Rees matrix semigroup $M=M(Q, X, Y, P)$ divides $X^{l} \times\left(Q w Y^{r}\right)$, where $X^{l}$ is a semigroup of left zeros, and $Y^{r}$ a semigroup of right zeros. In our case, $Q=C(m)$ is an Abelian group of exponent $m$ and therefore $\left(Q w Y^{r}\right) \in \mathbf{A}_{\mathbf{m}} \mathbf{w} \mathbf{R}_{\mathbf{1}}$. On the other hand, $X^{l} \in \mathbf{A}_{\mathbf{m}} \mathbf{w} \mathbf{R}_{\mathbf{1}}$ by Lemma 2.28. Consequently, the direct product of these semigroups, as well as the semigroup $S_{m}$, belong to $\mathbf{A}_{\mathbf{m}} \mathbf{w} \mathbf{R}_{\mathbf{1}}$. Proposition 2.31 is proved.

Proof of Theorem 2.1. Finite bases of identities of the wreath products of atoms of the lattice of semigroup varieties were found in Corollaries 2.5, 2.6, 2.8, 2.14, Lemmas 2.10, 2.15 and Propositions 2.17, 2.25, 2.31.

The fact that the varieties $\mathbf{S l w} \mathbf{A}_{\mathbf{n}}$ are essentially infinitely based was proved in [12. The fact that the wreath product of the group varieties has a finite basis follows from the results of the well-known book [6]. Indeed, the wreath product of varieties $\mathbf{A}_{\mathbf{m}} \mathbf{w} \mathbf{A}_{\mathbf{n}}$, being a metabelian variety, has a finite basis of identities [6, Theorem 36.11].

\section{The Calculation of the lattices of subvarieties OF THE WREATH PRODUCTS OF ATOMS}

In this section we prove Theorems 3.1 and 3.2, which yield the assertion of Theorem 1.2, that the lattice of subvarieties of the wreath product of atoms is finite. The proof actually consists of the proofs of several propositions corresponding to different cases of these theorems. Note that all the proofs given below use the information about bases of identities obtained in $\S 2$. As a rule, we also use the description of the identities 
of the wreath product of varieties $\mathbf{U w} \mathbf{V}$. In the simplest cases this description is not formulated explicitly.

Theorem 3.1. The cardinality of the lattice $\mathcal{L}(\mathbf{U}, \mathbf{V})$ in the case where $\mathbf{U}$ and $\mathbf{V}$ are atoms of the lattice of semigroup varieties is indicated in Table 1 .

TABLE 1.

$\begin{array}{llllll} & \mathbf{N}_{\mathbf{2}} & \mathbf{L}_{\mathbf{1}} & \mathbf{R}_{\mathbf{1}} & \mathbf{S l} & \mathbf{A}_{\mathbf{q}} \\ \mathbf{N}_{\mathbf{2}} & 4 & 4 & 4 & 4 & 4 \\ \mathbf{L}_{\mathbf{1}} & 4 & 4 & 4 & 4 & 4 \\ \mathbf{R}_{\mathbf{1}} & 10 & 8 & 5 & 10 & 4 \\ \mathbf{S l} & \text { finite } & 10 & \text { infinite } & \text { infinite } & \text { infinite } \\ \mathbf{A}_{\mathbf{p}} & 10 & 4 & 9 & 11 & 5 \text { for } p \neq q\end{array}$

Theorem 3.2. The cardinality of the lattice interval $\mathcal{I}(\mathbf{U}, \mathbf{V})=[\mathbf{U} \vee \mathbf{V}, \mathbf{U w} \mathbf{V}]$ in the case where $\mathbf{U}$ and $\mathbf{V}$ are atoms of the lattice of semigroup varieties is indicated in Table 2 .

TABLE 2 .

$\begin{array}{llllll} & \mathbf{N}_{\mathbf{2}} & \mathbf{L}_{\mathbf{1}} & \mathbf{R}_{\mathbf{1}} & \mathbf{S l} & \mathbf{A}_{\mathbf{q}} \\ \mathbf{N}_{\mathbf{2}} & 1 & 1 & 1 & 1 & 1 \\ \mathbf{L}_{\mathbf{1}} & 1 & 1 & 1 & 1 & 1 \\ \mathbf{R}_{\mathbf{1}} & 3 & 2 & 3 & 3 & 1 \\ \mathbf{S l} & \text { finite } & 3 & \text { infinite } & \text { infinite } & \text { infinite } \\ \mathbf{A}_{\mathbf{p}} & 3 & 1 & 3 & 4 & 2 \text { for } p \neq q\end{array}$

\section{Proposition 3.3.}

1) If $\mathbf{U}=\mathbf{N}_{\mathbf{2}}$, or $\mathbf{U}=\mathbf{L}_{\mathbf{1}}$, or $\mathbf{U}=\mathbf{R}_{\mathbf{1}}$ and $\mathbf{V}=\mathbf{A}_{\mathbf{p}}$, or $\mathbf{U}=\mathbf{A}_{\mathbf{p}}$ and $\mathbf{V}=\mathbf{L}_{\mathbf{1}}$, then $|\mathcal{L}(\mathbf{U w} \mathbf{V})|=4$ and $|\mathcal{I}(\mathbf{U}, \mathbf{V})|=1$.

2) Furthermore, $\left|\mathcal{I}\left(\mathbf{R}_{\mathbf{1}}, \mathbf{L}_{\mathbf{1}}\right)\right|=2$ and $\left|\mathcal{L}\left(\mathbf{R}_{\mathbf{1}} \mathbf{w} \mathbf{L}_{\mathbf{1}}\right)\right|=8$.

Proof. This proposition covers all the cases of atoms of the lattice of semigroup varieties in which the wreath product of atoms coincides with their lattice join. Then the lattice $\mathcal{L}(\mathbf{U w} \mathbf{V})$ is well known (see, for example, 25]).

Proposition 3.4. The lattice $\mathcal{L}\left(\mathbf{R}_{1}^{2}\right)$ of subvarieties of the variety $\mathbf{R}_{\mathbf{1}}^{2}$ is five-element and coincides with the lattice depicted in Figure 1. In particular, $\left|\mathcal{I}\left(\mathbf{R}_{\mathbf{1}}, \mathbf{R}_{\mathbf{1}}\right)\right|=3$.

Proof. Indeed, let $\mathbf{U} \subset \mathbf{R}_{\mathbf{1}}^{2}$, that is, $I\left(\mathbf{R}_{\mathbf{1}}^{\mathbf{2}}\right) \subset I(\mathbf{U})$. Let $u=v \in I(\mathbf{U}) \backslash I\left(\mathbf{R}_{\mathbf{1}}^{\mathbf{2}}\right)$. Then $t_{2}(u) \neq t_{2}(v)$. Here, either the identity $x=x^{2}$ is true in $\mathbf{U}$, or the identity $u \equiv u_{1} y z=$ $v_{1} y_{1} z \equiv v$ is true, where $y_{1} \neq y$. In the first of these cases, $\mathbf{U} \subseteq \mathbf{R}_{\mathbf{1}}$. In the second case we have $y z=u_{1} y z=v_{1} y_{1} z=y_{1} z \in I(\mathbf{U})$. If in addition $y_{1}=z$, then $y z=z^{2}=x z$, where $x \neq y$. Thus, in any case we have $\mathbf{U} \subseteq \mathbf{R}_{\mathbf{1}} \vee \mathbf{N}_{\mathbf{2}}$.

Proposition 3.5. The lattice $\mathcal{L}\left(\mathbf{R}_{\mathbf{1}} \mathbf{w} \mathbf{N}_{\mathbf{2}}\right)$ of all subvarieties of the variety $\mathbf{R}_{\mathbf{1}} \mathbf{w} \mathbf{N}_{\mathbf{2}}$ is depicted in Figure 2. In particular, $\mathbf{R}_{\mathbf{1}} \mathbf{w} \mathbf{N}_{\mathbf{2}}=\mathbf{R}_{\mathbf{1}, \mathbf{3}}=\operatorname{var}\left\{x_{1} x_{2} z=y_{1} y_{2} z\right\}$. Furthermore, $\left|\mathcal{L}\left(\mathbf{R}_{\mathbf{1}} \mathbf{w} \mathbf{N}_{\mathbf{2}}\right)\right|=10$ and $\left|\mathcal{I}\left(\mathbf{R}_{\mathbf{1}}, \mathbf{N}_{\mathbf{2}}\right)\right|=3$. 


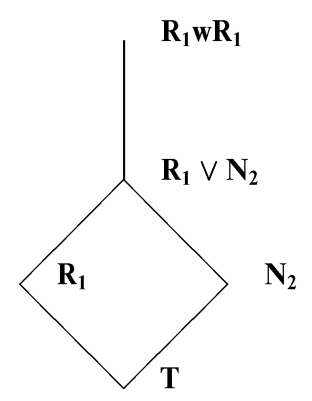

FiguRE 1.

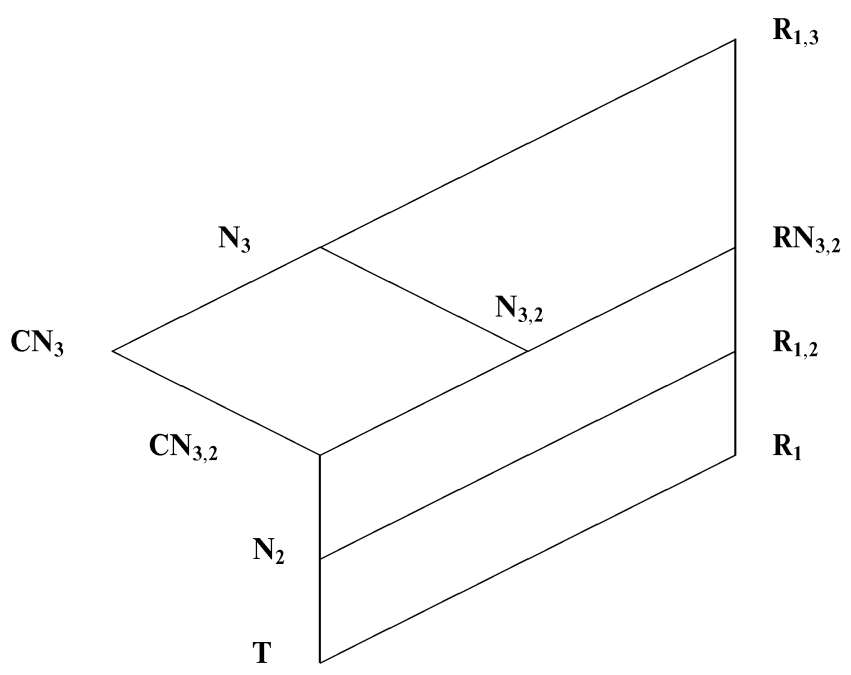

FiguRE 2.

In Figure 2 we use the following additional notation:

$$
\begin{aligned}
\mathbf{N}_{\mathbf{3}, \mathbf{2}} & =\operatorname{var}\left\{x^{2}=y_{1} y_{2} y_{3}\right\}, \\
\mathbf{C N}_{\mathbf{3}, \mathbf{2}} & =\operatorname{var}\left\{x^{2}=y_{1} y_{2} y_{3}, x y=y x\right\}, \\
\mathbf{C N}_{\mathbf{3}} & =\operatorname{var}\left\{y_{1} y_{2} y_{3}=z_{1} z_{2} z_{3}, x y=y x\right\}, \\
\mathbf{R N}_{\mathbf{3}, \mathbf{2}} & =\operatorname{var}\left\{z^{2}=y_{1} y_{2} z\right\}
\end{aligned}
$$

Proof. Let $\mathbf{U} \subset \mathbf{R}_{\mathbf{1 , 3}}=\operatorname{var}\left\{x_{1} x_{2} z=y_{1} y_{2} z\right\}$. Then $I\left(\mathbf{R}_{\mathbf{1 , 3}}\right) \subset I(\mathbf{U})$. Suppose that $u=v \in I(\mathbf{U}) \backslash I\left(\mathbf{R}_{1,3}\right)$. Then the identity $u=v$ does not satisfy either condition 1$)$ that either $u=v$ be a trivial identity or $|u|,|v| \geq 3$, or condition 2) that $t_{1}(u)=t_{1}(v)$ from the description of the identities of the set $I\left(\mathbf{R}_{\mathbf{1 , 3}}\right)$.

Case 1. Suppose that $t_{1}(u) \neq t_{1}(v)$. Then an identity of the form $u_{1} x=v_{1} y$, where $x \neq y$, is true in $\mathbf{U}$. We multiply this identity on the left by $x_{1} x_{2}$, where $x_{1}$ and $x_{2}$ are distinct variables that do not belong to the set $\{x, y\}$. By using the identity $x_{1} x_{2} z=$ $y_{1} y_{2} z$ we obtain the identity $x_{1} x_{2} x=y_{1} y_{2} y$. Thus, $\mathbf{U} \subseteq \operatorname{var}\left\{x_{1} x_{2} x=y_{1} y_{2} y\right\}=\mathbf{N}_{\mathbf{3}}$. All the subvarieties of the variety $\mathbf{N}_{\mathbf{3}}$ are known and can easily be calculated.

Case 2. Now suppose that the identity $u=v$ does not satisfy condition 1 ). We can assume in addition that $t_{1}(u)=t_{1}(v)$; otherwise case 1 can be applied to $\mathbf{U}$ and 
$\mathbf{U} \subseteq \mathbf{N}_{\mathbf{3}}$. Next, we can assume that then $|u| \leq 2$ and $|v| \leq 3$. In this case, the identity $z^{2}=z^{3}=y_{1} y_{2} z$ is automatically true in $\mathbf{U}$. Consequently, $\mathbf{U} \subseteq \mathbf{R N}_{\mathbf{3}, \mathbf{2}}$.

It is easy to see that the variety $\mathbf{W}_{\mathbf{1}}=\mathbf{S l w} \mathbf{L}_{\mathbf{1}}$ contains the variety $\operatorname{var} P^{\prime}$ generated by the three-element semigroup $P^{\prime}=\{0, e, a\}$ in which $a e=a, e^{2}=e$, and all the other products are equal to 0 . The semigroup $P^{\prime}$ is well known in the literature (see the survey [17, as well as [3, 8]). It is known that the variety var $P^{\prime}$ has the following basis of identities:

$$
x y=x y^{2}, \quad x^{2} y=y^{2} x .
$$

The proposition is proved.

Proposition 3.6. The lattice $\mathcal{L}\left(\mathbf{S l w}_{\mathbf{1}}\right)$ is depicted in Figure 3, In particular,

$$
\left|\mathcal{L}\left(\mathbf{S l w}_{\mathbf{1}}\right)\right|=10 \quad \text { and } \quad \mathcal{I}\left(\mathbf{S l}, \mathbf{L}_{\mathbf{1}}\right)=3 .
$$

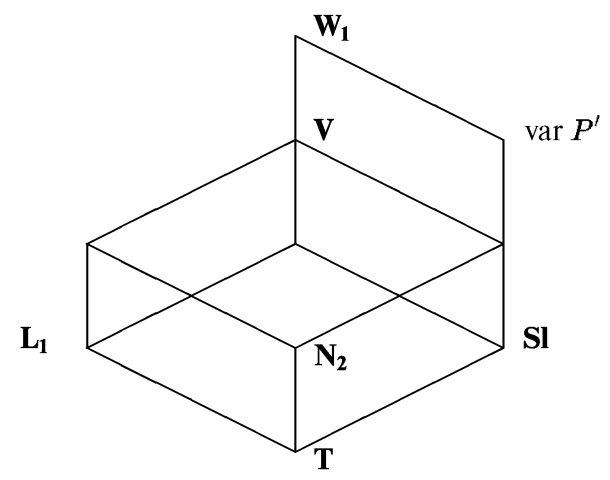

FIGURE 3.

In Figure 3 we use the following additional notation:

$$
\mathbf{W}_{1}=\mathbf{S l w L}_{\mathbf{1}}, \quad \mathbf{V}=\mathbf{S l} \vee \mathbf{L}_{\mathbf{1}} \vee \mathbf{N}_{\mathbf{2}} .
$$

Proof. By Theorem 1.1, $\mathbf{W}_{\mathbf{1}}=\operatorname{var}\left\{z x=z x^{2}, z y x=z x y\right\}$. On the other hand, it is known that $\mathbf{V}=\operatorname{var}\left\{z x=z x^{2}, z y x=z x y, z x=z^{2} x\right\}$. By using Lemma 18 in [4] it is easy to establish that the variety $\mathbf{W}_{\mathbf{1}}$ contains 5 subdirectly indecomposable semigroups: namely, the two-element semigroups $L$ of left zeros, $N$ with zero multiplication, the semilattice $U_{2}$, the semigroup $L^{0}$ that is equal to $L$ with an externally adjoint zero, and the semigroup $P^{\prime}$ defined above. As is well known, each variety of semigroups is generated by its subdirectly indecomposable semigroups. Consequently, it is easy to list all the subvarieties in $\mathbf{W}_{\mathbf{1}}$ :

$$
\begin{aligned}
\mathbf{L}_{\mathbf{1}} & =\operatorname{var} L, \\
\mathbf{N}_{\mathbf{2}} & =\operatorname{var} N, \\
\mathbf{S l} & =\operatorname{var} U_{2}, \\
\mathbf{L}_{\mathbf{1}} \vee \mathbf{N}_{\mathbf{2}} & =\operatorname{var}\{L, N\}, \\
\mathbf{N}_{\mathbf{2}} \vee \mathbf{S} \mathbf{l} & =\operatorname{var}\left\{N, U_{2},\right\}, \\
\mathbf{L}_{\mathbf{1}} \vee \mathbf{S} \mathbf{l} & =\operatorname{var}\left\{L^{0}\right\}=\operatorname{var}\left\{L^{0}, U_{2}, N\right\}, \\
\mathbf{V}=\mathbf{L}_{\mathbf{1}} \vee \mathbf{S} \mathbf{l} \vee \mathbf{N}_{\mathbf{2}} & =\operatorname{var}\left\{L^{0}, U_{2}, L, N\right\}=\operatorname{var}\left\{L^{0}, N\right\}, \\
\operatorname{var} P^{\prime} & =\operatorname{var}\left\{P^{\prime}, U_{2}, N\right\}, \\
\mathbf{W}_{\mathbf{1}} & =\operatorname{var}\left\{P^{\prime}, L^{0}\right\}=\operatorname{var}\left\{P^{\prime}, L^{0}, N, U_{2}, L\right\} .
\end{aligned}
$$


Corollary 3.7. The lattice $\mathcal{L}\left(\mathbf{R}_{\mathbf{1}} \mathbf{w S I}\right)$ is depicted in Figure 4 . In particular,

$$
\left|\mathcal{L}\left(\mathbf{R}_{\mathbf{1}} \mathbf{w} \mathbf{S l}\right)\right|=10 \quad \text { and } \quad \mathcal{I}\left(\mathbf{R}_{\mathbf{1}}, \mathbf{S l}\right)=3 .
$$

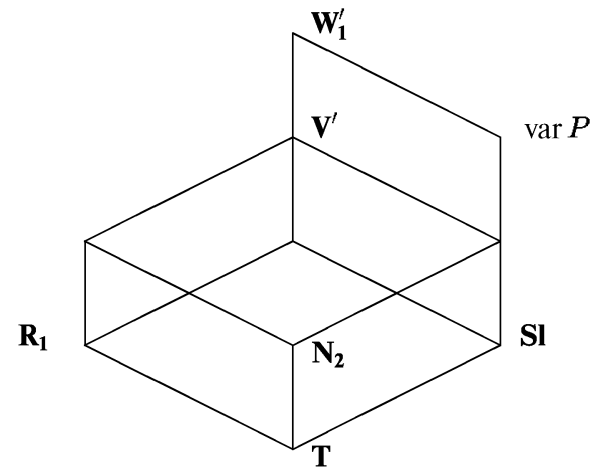

FiguRE 4.

Proof. The proof follows in an obvious fashion from the duality of the bases of identities for the varieties $\mathbf{S l w}_{\mathbf{1}}$ and $\mathbf{R}_{\mathbf{1}} \mathbf{w S l}$, which in turn follows from Corollary 2.4 and Lemma 2.10.

Proposition 3.8. The lattice $\mathcal{L}\left(\mathbf{S l w N}_{2}\right)$ is finite.

Proof. In Corollary 2.13 conditions are stated under which an identity $u=v$ belongs to the set $I\left(\mathbf{S l w} \mathbf{N}_{\mathbf{2}}\right)$; namely, it is necessary and sufficient that the following three conditions hold: 1) either $u=v$ is a trivial identity or $|u|,|v| \geq 3$;2) $h_{2}(u)=h_{2}(v)$; 3 ) the identity obtained from $u=v$ by replacing the common beginning $h_{2}(u)=h_{2}(v)$ by the subword $z_{1} z_{2}$, where $z_{1}, z_{2} \notin c(u v)$, and having the form

$$
z_{1} z_{2} u_{1}=z_{1} z_{2} v_{1}
$$

satisfies the property $c\left(u_{1}\right)=c\left(v_{1}\right)$. Now suppose that $\mathbf{U} \subset \mathbf{W}=\mathbf{S} \mathbf{w} \mathbf{w} \mathbf{N}_{\mathbf{2}}$. Then there exists an identity $u=v \in I(\mathbf{U}) \backslash I(\mathbf{W})$. It is obvious that for the identity $u=v$ at least one of the three conditions of Lemma 2.13 is violated. We consider two cases.

Case 1. Suppose that some identity $u=v$ with the condition $c(u) \neq c(v)$ is true in $\mathbf{U}$. We multiply the identity $u=v$ on the left by the factor $z_{1} z_{2}$, where $z_{1}, z_{2} \notin c(u v)$, and obtain the identity $z_{1} z_{2} u=z_{1} z_{2} v$, in which there is a letter $y$ that occurs in exactly one of the words $z_{1} z_{2} u$ or $z_{1} z_{2} v$. For definiteness suppose that $y \in c(u) \backslash c(v)$. In this identity we set $\phi\left(z_{i}\right)=z_{i}(i=1,2), \phi(y)=y, \phi(t)=x$ for any $t \in X \backslash\left\{z_{1}, z_{2}, y\right\}$. As a result we obtain the identity $z_{1} z_{2} y x=z_{1} z_{2} x$, which is true in $\mathbf{U} \subset \mathbf{W}$ and which in $\mathbf{W}$ easily implies the identity

$$
z_{1} z_{2} y=z_{1} z_{2} x .
$$

It is known that the identity (3.3) defines the variety $\mathbf{L}_{\mathbf{2 , 3}}$, which is an idempotent of the monoid $M V$ of all semigroup varieties. As already noted in [15], the lattices $\mathcal{L}\left(\mathbf{L}_{\mathbf{j}, \mathbf{m}}\right)$ are finite for any $m \geq 1, j \leq m$.

Case 2. Now suppose that $I(\mathbf{U})$ does not contain identities with the condition $c(u) \neq$ $c(v)$, that is, all the identities in $I(\mathbf{U})$ are homotypic. We can assume in what follows 
that the identity $u=v$ is homotypic and the words $u$ and $v$ in it are reduced in the set $I(\mathbf{W})$, that is, each of them has one of the following forms:

$$
\begin{gathered}
z, z^{2} x_{1} \ldots x_{k}, z^{3} x_{1} \ldots x_{k}, z_{1} z_{2} x_{1} \ldots x_{k}, z_{1} z_{2} z_{1} x_{1} \ldots x_{k}, \\
z_{1} z_{2}^{2} x_{1} \ldots x_{k}, z_{1} z_{2}^{2} z_{1} x_{1} \ldots x_{k} \quad(k \geq 0) .
\end{gathered}
$$

If $|u|,|v| \geq 3$, then the identity $u=v$ has the form

$$
u \equiv w\left(z_{1}, z_{2}\right) x_{1} \ldots x_{k}=w_{1}\left(z_{3}, z_{4}\right) x_{1}^{\prime} \ldots x_{l}^{\prime} \equiv v,
$$

where $|w|,\left|w_{1}\right| \leq 4$. Furthermore, if $\left\{z_{1}, z_{2}\right\} \cap\left\{z_{3}, z_{4}\right\}=\varnothing$, then the letters $z_{1}, z_{2}$ are contained in the set $\left\{x_{1}^{\prime}, \ldots, x_{l}^{\prime}\right\}$, while $z_{3}, z_{4}$ are contained in the set $\left\{x_{1}, \ldots, x_{k}\right\}$ since we have assumed that the identity $u=v$ is homotypic. Obviously, the identity (3.4) is $\mathbf{W}$-equivalent to an identity of the form

$$
u^{\prime} \equiv w\left(z_{1}, z_{2}\right) z_{3} z_{4} x=w_{1}\left(z_{3}, z_{4}\right) z_{1} z_{2} x \equiv v^{\prime} .
$$

Here it is possible that the letter $x$ is absent in (3.5) if (3.4) depends only on four letters; but in any case, $\left|u^{\prime}\right|,\left|v^{\prime}\right| \leq 7$, and (3.5) depends on at most 5 letters.

Consequently, up to $\mathbf{W}$-equivalence there are at most finitely many homotypic identities $u=v$ that satisfy $|u|,|v| \geq 3$. Obviously, up to $\mathbf{W}$-equivalence there are also finitely many such identities that satisfy $|u| \leq 2$.

Every subvariety $\mathbf{U} \subseteq \mathbf{W}$ containing $\mathbf{S l}$ can be defined by some set of homotypic identities. Since there are finitely many such sets, the lattice $\mathcal{L}\left(\mathbf{S l w} \mathbf{N}_{\mathbf{2}}\right)$ is finite.

The description of the lattice $\mathcal{L}\left(\mathbf{A}_{\mathbf{p}} \mathbf{w} \mathbf{R}_{\mathbf{1}}\right)$ follows from the description of all subvarieties of the variety of completely simple semigroups over Abelian groups (see [7, Theorem 4]) and from Proposition 2.30, which indicates a basis of identities of the variety $\mathbf{A}_{\mathbf{p}} \mathbf{w} \mathbf{R}_{\mathbf{1}}$ and a completely simple semigroup $S_{p}$ generating this variety.

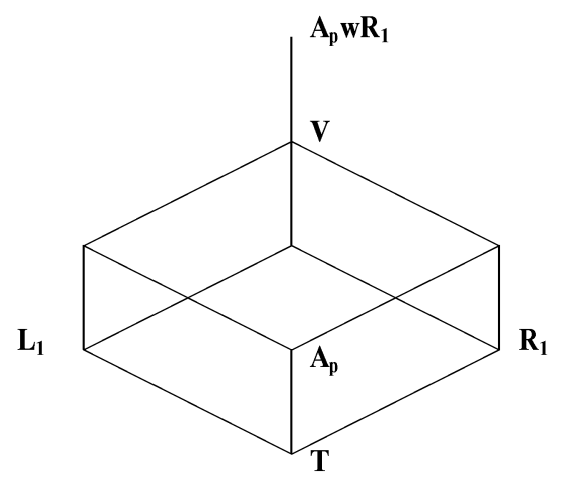

FIGURE 5 .

Proposition 3.9. The lattice $\mathcal{L}\left(\mathbf{A}_{\mathbf{p}} \mathbf{w} \mathbf{R}_{\mathbf{1}}\right)$ of all subvarieties of the wreath product $\mathbf{A}_{\mathbf{p}} \mathbf{w} \mathbf{R}_{\mathbf{1}}$ is depicted in Figure 5. In particular, $\left|\mathcal{L}\left(\mathbf{A}_{\mathbf{p}} \mathbf{w} \mathbf{R}_{\mathbf{1}}\right)\right|=9$ and $\left|\mathcal{I}\left(\mathbf{A}_{\mathbf{p}}, \mathbf{R}_{\mathbf{1}}\right)\right|=3$.

In Figure [5] we denote the variety $\mathbf{A}_{\mathbf{p}} \vee \mathbf{R}_{\mathbf{1}} \vee \mathbf{L}_{\mathbf{1}}$ by $\mathbf{V}$. For completeness we point out that from the description of the identities of the atoms of the lattice of semigroup varieties it easily follows that

$$
\begin{aligned}
\mathbf{A}_{\mathbf{p}} \vee \mathbf{L}_{\mathbf{1}} & =\operatorname{var}\left\{x=x y^{p}, \quad z x y=z y x\right\}, \\
\mathbf{A}_{\mathbf{p}} \vee \mathbf{R}_{\mathbf{1}} & =\operatorname{var}\left\{x=x y^{p}, \quad x y z=y x z\right\}, \\
\mathbf{A}_{\mathbf{p}} \vee \mathbf{R}_{\mathbf{1}} \vee \mathbf{L}_{\mathbf{1}} & =\operatorname{var}\left\{x=x y^{p}, \quad z x y t=z y x t, \quad x z=x y^{p} z\right\} .
\end{aligned}
$$


The description of the bases of identities for these varieties which follows from [7, Theorem 4] is more complicated. The fact that these are the same varieties follows from the following remark, which can be easily verified.

Remark 3.10. The following equalities hold:

a) $\mathbf{A}_{\mathbf{p}} \vee \mathbf{L}_{\mathbf{1}}=\operatorname{var}\left\{x=x(y x)^{p}, x^{2} y x=x y x^{2}, x^{p} y^{p}=x^{p}\right\}$;

b) $\mathbf{A}_{\mathbf{p}} \vee \mathbf{R}_{\mathbf{1}}=\operatorname{var}\left\{x=x(y x)^{p}, x^{2} y x=x y x^{2}, x^{p} y^{p}=y^{p}\right\}$;

c) $\mathbf{A}_{\mathbf{p}} \vee \mathbf{R}_{\mathbf{1}} \vee \mathbf{L}_{\mathbf{1}}=\operatorname{var}\left\{x=x(y x)^{p}, x^{2} y x=x y x^{2}, x^{p} y^{p}=(x y)^{p}\right\}$.

To describe the lattice $\mathcal{L}\left(\mathbf{A}_{\mathbf{p}} \mathbf{w} \mathbf{N}_{\mathbf{2}}\right)$ we first give a description of the identities of the variety $\mathbf{A}_{\mathbf{m}} \mathbf{w} \mathbf{N}_{2}$.

Proposition 3.11. The identities of $\mathbf{A}_{\mathbf{m}} \mathbf{w} \mathbf{N}_{\mathbf{2}}$ are given by $I\left(\mathbf{A}_{\mathbf{m}} \mathbf{w} \mathbf{N}_{\mathbf{2}}\right)=\{u=v$ : 1) either $u=v$ is a trivial identity or $|u|,|v| \geq 2$; 2) for any $x$ in $X,|u|_{x} \equiv|v|_{x}(\bmod m)$; 3) $\left.h_{2}(u)=h_{2}(v)\right\}$.

Proof. The first two conditions follow from the inclusion $\mathbf{A}_{\mathbf{m}} \mathbf{w} \mathbf{N}_{\mathbf{2}} \supseteq \mathbf{A}_{\mathbf{m}} \vee \mathbf{N}_{\mathbf{2}}$. Next, let $\mathbf{V}_{\mathbf{m}}=\mathbf{A}_{\mathbf{m}} \mathbf{w} \mathbf{N}_{\mathbf{2}}$ and let $u=v \in I\left(\mathbf{V}_{\mathbf{m}}\right)$. This identity induces the equality

$$
f_{1}(1) f_{2}\left(p_{1}\right) \ldots f_{k}\left(p_{1} \ldots p_{k-1}\right)=g_{1}(1) g_{2}\left(q_{1}\right) \ldots g_{l}\left(q_{1} \ldots q_{l-1}\right)
$$

for any functions $f_{1}, \ldots, f_{k}, g_{1}, \ldots, g_{l} \in S^{R^{1}}$, where $S \in \mathbf{A}_{\mathbf{m}}$ and $R \in \mathbf{N}_{\mathbf{2}}$. By condition 1) we can assume that $k, l \geq 2$. Taking into account that $R \in \mathbf{N}_{2}$ we can rewrite equality (3.9) in the form

$$
f_{1}(1) f_{2}\left(p_{1}\right) f_{3}(0) \ldots f_{k}(0)=g_{1}(1) g_{2}\left(q_{1}\right) g_{3}(0) \ldots g_{l}(0) .
$$

According to Remark 2.2, the last equality induces the identity

$$
x_{1} x_{2} \tilde{u}_{1}=y_{1} y_{2} \tilde{v}_{1}
$$

in $\mathbf{A}_{\mathbf{m}}$. If we choose $p_{1} \neq 0, q_{1} \neq 0$, which is possible in the case $|R| \geq 2$, then in (3.11) we have $x_{1}, x_{2}, y_{1}, y_{2} \notin c\left(\tilde{u}_{1} \tilde{v}_{1}\right)$. Consequently, if $h_{1}(u) \neq h_{1}(v)$ or $h_{1}(u)=h_{1}(v)$ but $h_{2}(u) \neq h_{2}(v)$, then the identity (3.11) violates condition 2), but condition 2) is true in $\mathbf{A}_{\mathbf{m}}$. Thus, condition 3) is also satisfied for an identity $u=v \in I\left(\mathbf{V}_{\mathbf{m}}\right)$.

Conversely, any identity $u=v$ satisfying conditions 1)-3) of Proposition 3.11 can be derived from the basis (2.11) indicated in Lemma 2.15 and, consequently, is true in $\mathbf{V}_{\mathbf{m}}$. Indeed, if an identity $u=v$ satisfies conditions 1) -3 ) and is non-trivial, then by using the identities $x y z^{m}=x y$ and $z_{1} z_{2} x y=z_{1} z_{2} y x$ we can reduce any word $u$ of length greater than 1 to the form

$$
u^{\prime} \equiv h_{2}(u) y_{1}^{a_{1}} \ldots y_{k}^{a_{k}},
$$

where $0 \leq a_{i} \leq m-1$. Suppose that the word $v$ in the identity $u=v$ is reduced to the form

$$
v^{\prime} \equiv h_{2}(v) y_{1}^{b_{1}} \ldots y_{k}^{b_{k}},
$$

where $0 \leq b_{i} \leq m-1$. If $y_{1}, \ldots, y_{k} \notin c\left(h_{2}(u)\right)$, then by condition 3$)$ this implies that $b_{i}=a_{i}(i=1, \ldots, k)$. Let $y_{i} \in c\left(h_{2}(u)\right)=\{x\}$. Then

$$
x^{2} y_{1}^{a_{1}} \ldots y_{i-1}^{a_{i-1}} x^{a_{i}} \ldots y_{k}^{a_{k}}=x^{2} y_{1}^{b_{1}} \ldots y_{i-1}^{b_{i-1}} x^{b_{i}} \ldots y_{k}^{b_{k}} \text {. }
$$

Hence we obtain that $a_{i}+2 \equiv b_{i}+2(\bmod m)$. Thus, the identity $x^{2} y x^{m-1}=x^{m+1} y$ holds in $\mathbf{V}_{\mathbf{m}}$. But this identity can be derived from the identities of the basis (2.11). A similar argument is possible if $h_{2}(u) \equiv x y$. The variables in the reduced word (3.12) are ordered as follows: $h_{1}(u), c\left(h_{2}(u)\right) \backslash h_{1}(u)$, and then according to the order in the alphabet $X$, where $X$ is a denumerable ordered alphabet. 
Proposition 3.12. The lattice $\mathcal{L}\left(\mathbf{A}_{\mathbf{p}} \mathbf{w} \mathbf{N}_{\mathbf{2}}\right)$ of all subvarieties of the wreath product $\mathbf{A}_{\mathbf{p}} \mathbf{w} \mathbf{N}_{\mathbf{2}}$ is depicted in Figure 6. In particular, $\left|\mathcal{L}\left(\mathbf{A}_{\mathbf{p}} \mathbf{w} \mathbf{N}_{\mathbf{2}}\right)\right|=10$ and $\left|\mathcal{I}\left(\mathbf{A}_{\mathbf{p}}, \mathbf{N}_{\mathbf{2}}\right)\right|=3$.

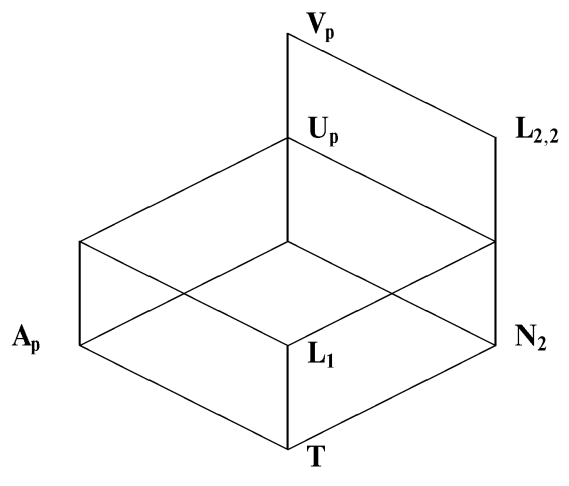

FIGURE 6.

In Figure 6. $\mathbf{U}_{\mathbf{p}}$ denotes the variety $\mathbf{A}_{\mathbf{p}} \vee \mathbf{L}_{\mathbf{1}} \vee \mathbf{N}_{\mathbf{2}}$, and $\mathbf{V}_{\mathbf{p}}$ the variety $\mathbf{A}_{\mathbf{p}} \mathbf{w} \mathbf{N}_{\mathbf{2}}$, and $\mathbf{L}_{\mathbf{2}, \mathbf{2}}=\operatorname{var}\left\{x_{1} x_{2} y=x_{1} x_{2}\right\}$.

Remark 3.13. The identities $z y x=z x y$ and $z x y^{p}=z x$ form a basis of identities of the variety $\mathbf{A}_{\mathbf{p}} \vee \mathbf{L}_{\mathbf{1}} \vee \mathbf{N}_{\mathbf{2}}$. Before proving Proposition 3.12 we introduce some notation and state two lemmas from [4]. Let

$$
\begin{gathered}
M_{1}=\operatorname{var}\left\{x y z=t^{2}=0, x y=y x\right\}, \\
M_{2}=\operatorname{var}\{x y=x y z\}, \quad M_{2}^{*}=\operatorname{var}\{x y=z x y\}, \\
M_{5}=\operatorname{var}\left\{x=x^{2}, x y=x y x\right\}, \quad M_{5}^{*}=\operatorname{var}\left\{x=x^{2}=0, y x=x y x\right\}, \\
M(q)=\operatorname{var}\left\{x=(x y)^{q} x, \quad x=x^{1+q}, x y x^{2}=x^{2} y x\right\},
\end{gathered}
$$

where $q$ is a prime.

Lemma 3.14 (4 Lemma 5]). If the identity $x y=(x y)^{n+1}(n \geq 1)$ is true in a variety $\mathbf{U}$, which does not contain the varieties $M_{1}, M_{2}, M_{2}^{*}, M_{5}, M_{5}^{*}$ or $M(q)$ for any prime $q$, then the identities $x^{n+1} y=x y=x y^{n+1}$ and $x y^{n} z^{n} t=x z^{n} y^{n} t$ are true in $\mathbf{U}$.

Lemma 3.15 (4, Lemma 16]). If the identities $x^{n+1} y=x y=x y^{n+1}$ and $x y^{n} z^{n} t=$ $x z^{n} y^{n} t$ are true in a variety $\mathbf{U}$, then the subdirectly indecomposable semigroups in $\mathbf{U}$ are contained in the set $\left\{L, L^{0}, R, R^{0}, U_{2}, N, G_{i}, G_{i}^{0}\right\}$, where the $G_{i}$ are subdirectly indecomposable groups in $\mathbf{U}$.

Proof of Proposition 3.12. Let $\mathbf{U} \subset \mathbf{V}_{\mathbf{p}}=\mathbf{A}_{\mathbf{p}} \mathbf{w} \mathbf{N}_{\mathbf{2}}$. Then $I\left(\mathbf{V}_{\mathbf{p}}\right) \subset I(\mathbf{U})$. Suppose that an identity $u=v$ belongs to $I(\mathbf{U}) \backslash I\left(\mathbf{V}_{\mathbf{p}}\right)$. Then at least one of the three conditions 1$)$, 2), 3) of Proposition 3.11 is violated for $u=v$.

Case 1. If $u=v$ does not satisfy condition 1 ), then the identity

$$
x=x^{1+p}
$$

is true in $\mathbf{U}$. Then in view of the basis of identities (2.13) for $m=p$ of the variety $\mathbf{V}_{\mathbf{p}}$, the chain of identities $z y x=z z^{p} y x=z z^{p} x y=z x y$ is true in $\mathbf{U}$. Consequently, $\mathbf{U} \subseteq \mathbf{A}_{\mathbf{p}} \vee \mathbf{L}_{\mathbf{1}}$.

Case 2. Suppose that $u=v$ does not satisfy condition 2). Then an identity of the form $x^{m}=x^{m+d}$, where $1 \leq d \leq p-1$, is true in $\mathbf{U}$. This fact and the identity $x^{2}=x^{2+p}$ imply that $x^{2}=x^{3} \in I(\mathbf{U})$. Then the chain of identities $z x=z x y^{p}=z x y^{p+1}=z x y$ is also true in $\mathbf{U}$. Thus, in this case, $\mathbf{U} \subseteq \operatorname{var}\{z x=z x y\}=\mathbf{L}_{\mathbf{2}, \mathbf{2}}$. The lattice $\mathcal{L}\left(\mathbf{L}_{\mathbf{2}, \mathbf{2}}\right)$ is well known and contains 5 elements. 
Case 3. In what follows we can already assume that conditions 1) and 2) hold. Suppose that condition 3) is violated for the identity $u=v$, that is, $h_{2}(u) \neq h_{2}(v)$. Obviously, $\mathbf{V}_{\mathbf{p}}$ satisfies the identity $x y=(x y)^{1+p}$, does not contain the two-element semigroup $R$ of right zeros, the two-element semigroup $L^{0}$ of left zeros with adjoint zero, or the four-element semigroup $Q=\{a, b, d, 0\}$ in which $a b=b a=d$ and all the other products are equal to zero. Furthermore, $R \in \mathbf{M}_{\mathbf{2}}^{*}, \mathbf{M}_{\mathbf{5}}^{*}, \mathbf{M}(\mathbf{q})$ and, consequently, the varieties $\mathbf{M}_{\mathbf{2}}^{*}, \mathbf{M}_{\mathbf{5}}^{*}, \mathbf{M}(\mathbf{q})$ are not contained in $\mathbf{V}_{\mathbf{p}}$. We have $L^{0} \in \mathbf{M}_{\mathbf{5}}, Q \in \mathbf{M}_{\mathbf{1}}$, and, consequently, $\mathbf{M}_{\mathbf{1}}$ and $\mathbf{M}_{\mathbf{5}}$ are not contained in $\mathbf{V}_{\mathbf{p}}$. If $\mathbf{U} \subset \mathbf{V}_{\mathbf{p}}$ and in $\mathbf{U}$ the condition $h_{2}(u) \neq h_{2}(v)$ is not satisfied for some identity $u=v$, then $\mathbf{M}_{\mathbf{2}}=\mathbf{L}_{\mathbf{2}, \mathbf{2}}$ is not contained in $\mathbf{U}$. By Lemmas 3.14 and 3.15, in this case the variety $\mathbf{U}$ can contain only three subdirectly indecomposable semigroups $\mathbf{L}, \mathbf{N}, \mathbf{C}(\mathbf{p})$. Consequently, in this case, $\mathbf{U} \subseteq \mathbf{U}_{\mathbf{p}}=\mathbf{L}_{\mathbf{1}} \vee \mathbf{N}_{\mathbf{2}} \vee \mathbf{A}_{\mathbf{p}}$.

Proposition 3.16. The lattice $\mathcal{L}\left(\mathbf{A}_{\mathbf{p}} \mathbf{w S l}\right)$ of all subvarieties of the wreath product $\mathbf{A}_{\mathbf{p}} \mathbf{w S l}$ is depicted in Figure $\mathbf{7}$. In particular, $\left|\mathcal{L}\left(\mathbf{A}_{\mathbf{p}} \mathbf{w S l}\right)\right|=11$ and $\left|\mathcal{I}\left(\mathbf{A}_{\mathbf{p}}, \mathbf{S l}\right)\right|=4$.

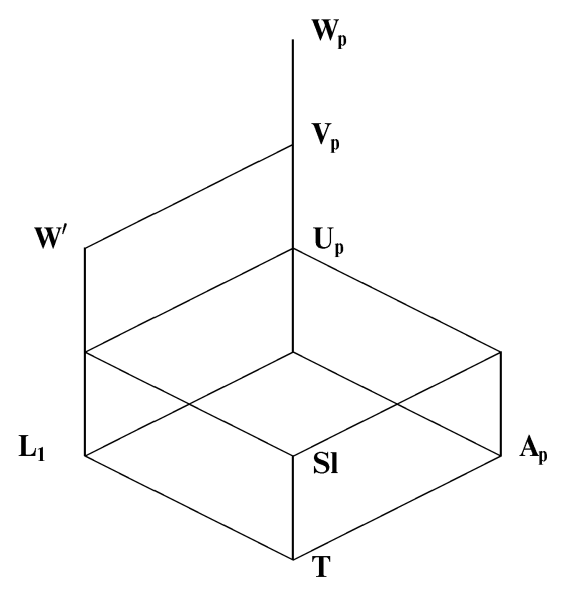

FIGURE 7.

In Figure 7 we denote by $\mathbf{W}_{\mathbf{p}}$ the variety $\mathbf{A}_{\mathbf{p}} \mathbf{w S l}$, by $\mathbf{U}_{\mathbf{p}}$ the variety $\mathbf{A}_{\mathbf{p}} \vee \mathbf{L}_{\mathbf{1}} \vee \mathbf{S l}$, and set

$$
\begin{aligned}
\mathbf{V}_{\mathbf{p}} & =\operatorname{var}\left\{x=x^{1+p}, x y x=x^{2} y\right\}, \\
\mathbf{W}^{\prime} & =\operatorname{var}\left\{x=x^{2}, x y x=x y\right\} .
\end{aligned}
$$

To prove Proposition 3.16 we use the results of $\underline{32}$. We recall some definitions and introduce notation. We denote by $\mathbf{B}$ the variety of all bands, by $\mathbf{R e}$ the variety of rectangular bands, by $\mathbf{C S}$ the variety of all completely simple semigroups in the signature $\left\langle\cdot{ }^{-1}\right\rangle$, by BG the variety of all bands of groups in the same signature. Let $L G_{n}=\langle a, f$ : $\left.a=a^{1+n}, f^{2}=f, a^{n} f=f, f a=a\right\rangle$. The semigroup $L G_{n}$ is an ideal extension of the semigroup of left zeros by the cyclic group of order $n$. The semigroup $R G_{n}$ is defined as the right-hand analogue of the semigroup $L G_{n}$. By Theorem 1 in $[32$, a variety $\mathbf{V}$ of completely regular semigroups consists of bands of groups if and only if the semigroups $L G_{n}$ and $R G_{n}$ do not belong to $\mathbf{V}$ for any integer $n, n \geq 2$.

Lemma 3.17. The varieties $\mathbf{W}_{\mathbf{p}}$ and $\operatorname{var} L G_{p}$ satisfy $\mathbf{W}_{\mathbf{p}}=\operatorname{var} L G_{p}$.

Proof. It is easy to verify that the identities (2.20) and (2.21) are true in $L G_{p}$. Therefore, $L G_{p} \in \mathbf{W}_{\mathbf{p}}$. By Proposition 2.25, this implies their sets of identities satisfy the inclusion $I\left(\mathbf{W}_{\mathbf{p}}\right) \subseteq I\left(L G_{p}\right)$. To prove the reverse inclusion, suppose that an identity $u=v$ does 
not belong to $I\left(\mathbf{W}_{\mathbf{p}}\right)$. Using the description of such identities obtained in Lemma 2.20 one can easily establish, as was done in the proof of Proposition 2.25, that the identity $u=v$ is false in $I\left(L G_{p}\right)$, and therefore, $I\left(L G_{p}\right) \subseteq I\left(\mathbf{W}_{\mathbf{p}}\right)$. This proves the lemma.

Proof of Proposition 3.16. If $\mathbf{V} \subseteq \mathbf{W}^{\prime}$ and $\mathbf{V} \neq \mathbf{W}^{\prime}$, then $\mathbf{V}$ is a variety of bands of groups by Lemma 3.17 and Theorem 1 in 32. Note that in the case of periodic semigroups the notion of "a variety of semigroups" coincides with the notion of "a variety of completely regular semigroups". The variety $\mathbf{B G}_{\mathbf{p}}$ of all bands of groups of exponent $p$ is described by the identity

$$
\left(x^{p} y^{p}\right)^{p}=(x y)^{p},
$$

while the identity of condition 2) of Theorem 2 in 32 takes the form

$$
(x y)^{p} x^{p}(z x)^{p}=(x y x z x)^{p} .
$$

It is easy to verify that the variety $\mathbf{V}_{\mathbf{p}}=\mathbf{W}_{\mathbf{p}} \cap \mathbf{B G}$ is the largest variety of bands of groups contained in $\mathbf{W}_{\mathbf{p}}$. Furthermore, $\mathbf{V}_{\mathbf{p}}$ satisfies the identity (3.16). By Theorem 2 in [32, $\quad \mathbf{V}_{\mathbf{p}}$ is contained in the lattice join of the variety $\mathbf{C S}$ of all completely simple semigroups and the variety of all bands $\mathbf{B}$. Proposition 1 in 32 gives a description of the structure of the lattice of all subvarieties of $\mathbf{V}_{\mathbf{p}}$.

Proposition 3.18. The lattices $\mathcal{L}(\mathbf{S l w S l}), \mathcal{L}\left(\mathbf{S l w} \mathbf{R}_{\mathbf{1}}\right)$, and $\mathcal{L}\left(\mathbf{S l w} \mathbf{A}_{\mathbf{n}}\right)$ and the corresponding lattice intervals $\mathcal{I}(\mathbf{S l}, \mathbf{S l}), \mathcal{I}\left(\mathbf{S l}, \mathbf{R}_{\mathbf{1}}\right)$, and $\mathcal{I}\left(\mathbf{S l}, \mathbf{A}_{\mathbf{n}}\right)$, where $n$ is a positive integer greater than or equal to 2 , are infinite.

Proof. Consider the lattice of subvarieties of the variety $\mathbf{A}_{\mathbf{2}, \mathbf{1}}=\operatorname{var}\left\{x^{2}=x^{3}, x y=y x\right\}$. First of all we note that the lattice $\mathcal{L}\left(\mathbf{A}_{\mathbf{2}, \mathbf{1}}\right)$ has long been known and is depicted in Figure 8 (see, for example, the survey [25). In Figure 8 we also use the following notation: $\mathbf{W}_{\mathbf{0}}$ is the variety $\operatorname{var}\left\{x^{2}=x^{3}, x y=y x, x^{2} y=x y^{2}\right\}$ and $\mathbf{V}_{\mathbf{0}}$ is the variety $\operatorname{var}\left\{x^{2} y=x^{2}, x y=y x\right\}$. Furthermore, the $k$-th term in the lattice interval $\left[\mathbf{T}, \mathbf{V}_{\mathbf{0}}\right]$ is the variety $\mathbf{V}_{\mathbf{k}}=\operatorname{var}\left\{x y=y x, x^{2}=x^{3}, x_{1} \ldots x_{k}=y_{1} \ldots y_{k}\right\}$ and the $k$-th term in the lattice interval $\left[\mathbf{S} \mathbf{l}, \mathbf{W}_{\mathbf{0}}\right]$ is the variety $\mathbf{W}_{\mathbf{k}}=\operatorname{var}\left\{x y=y x, x^{2}=x^{3}, x_{1} \ldots x_{k}=x_{1} \ldots x_{k}^{2}\right\}$.

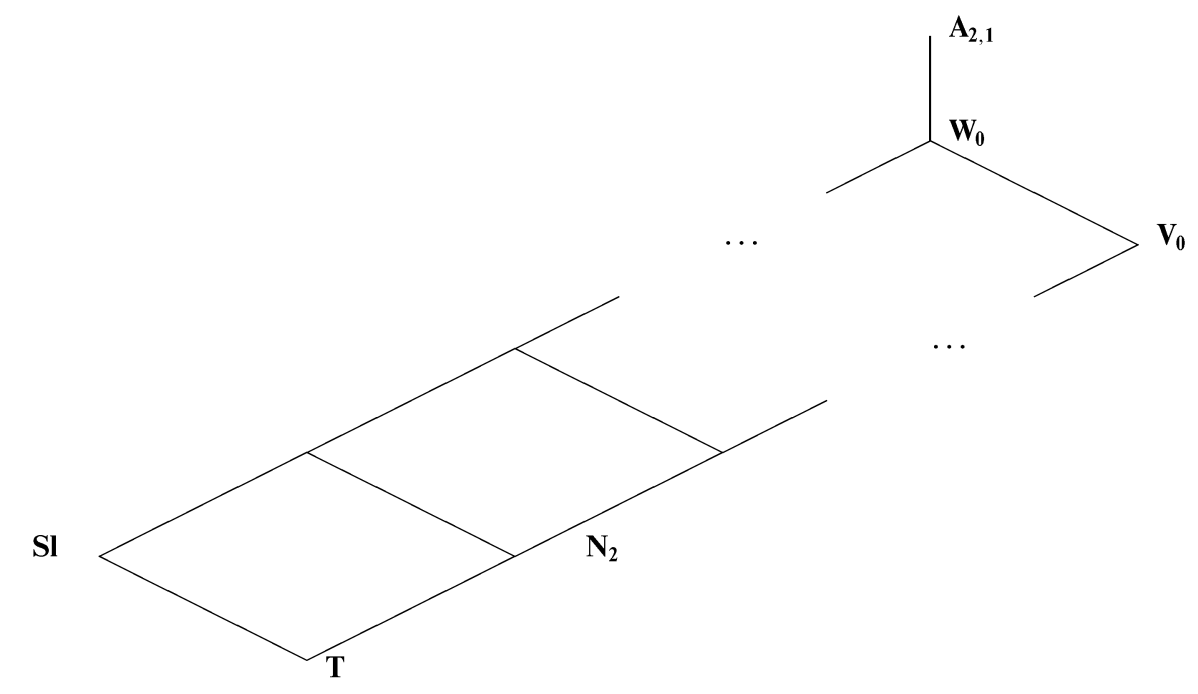

FigURE 8. 
It can be verified that $\mathbf{A}_{\mathbf{2}, \mathbf{1}} \subset \mathbf{S l w S}$, since from the basis of identities for the variety $\mathbf{A}_{\mathbf{2}, \mathbf{1}}$ it is easy to derive all the identities of the basis for the variety SlwSl that are indicated in Proposition 2.17. Similarly, it can be shown that $\mathbf{W}_{\mathbf{0}} \subset \mathbf{S l w} \mathbf{R}_{\mathbf{1}}$. Finally, in [12] it was proved that the Brandt six-element monoid $B_{2}^{1}$ belongs to the variety Slw $\mathbf{A}_{\mathbf{n}}$, where $n \geq 2$ is a positive integer. Hence we easily obtain the inclusions $\operatorname{Slw}_{\mathbf{w}} \mathbf{A}_{\mathbf{n}} \supset$ $\operatorname{var} B_{2}^{1} \supset \operatorname{var} B_{2}$. It is known that

$$
\operatorname{var} B_{2}=\operatorname{var}\left\{x^{2}=x^{3}, x y x=x y x y x, x^{2} y^{2}=y^{2} x^{2}\right\} .
$$

Again, as above, from the basis of identities for the variety $\mathbf{W}_{\mathbf{0}}$ one can easily derive all the identities of the basis for the variety var $B_{2}$ indicated above. Indeed, let

$$
B\left(\mathbf{W}_{\mathbf{0}}\right)=\left\{x^{2}=x^{3}, x y=y x, x^{2} y=x y^{2}\right\} .
$$

Then the first identity in the basis of identities of $B\left(\operatorname{var} B_{2}\right)$ occurs in the basis of $B\left(\mathbf{W}_{\mathbf{0}}\right)$, the third identity is a consequence of the commutativity law, and the second has the following inference chain:

$$
x y x=x^{2} y=x y^{2}=x y^{3}=x^{2} y^{2}=x^{3} y^{2}=x y x y x .
$$

Consequently, $I\left(\operatorname{var} B_{2}\right) \subseteq I\left(\mathbf{W}_{\mathbf{0}}\right)$, and hence, $\mathbf{W}_{\mathbf{0}} \subseteq$ var $B_{2}$. Thus, all the three lattices contain the infinite lattice interval $\left[\mathbf{S} \mathbf{l}, \mathbf{W}_{\mathbf{0}}\right]$. Hence the lattice intervals $\mathcal{I}(\mathbf{S l}, \mathbf{S l})$, $\mathcal{I}\left(\mathbf{S l}, \mathbf{R}_{\mathbf{1}}\right)$ and $\mathcal{I}\left(\mathbf{S l}, \mathbf{A}_{\mathbf{n}}\right)$ for any positive integer $n \geq 2$ are also infinite. Indeed, it is easy to verify that an identity $u=v$ belongs to the set of identities $I\left(\mathbf{W}_{\mathbf{k}}\right)$ if and only if it has the following two properties:

1) $c(u)=c(v)$;

2) $\left[|u| \geq k\right.$ or there exists a letter $x$ such that $\left.|u|_{x} \geq 2\right]$ and $[|v| \geq k$ or there exists a letter $y$ such that $\left.|v|_{y} \geq 2\right]$.

To prove that the lattice interval $\mathcal{I}\left(\mathbf{S l}, \mathbf{R}_{\mathbf{1}}\right)$ is infinite we note that the varieties $\mathbf{W}_{\mathbf{k}}^{\prime}=\mathbf{W}_{\mathbf{k}} \vee \mathbf{R}_{\mathbf{1}}$ satisfy $I\left(\mathbf{W}_{\mathbf{k}}^{\prime}\right)=I\left(\mathbf{W}_{\mathbf{k}}\right) \cap I\left(\mathbf{R}_{\mathbf{1}}\right)$. Hence we can observe that an identity $u=v$ belongs to the set of identities $I\left(\mathbf{W}_{\mathbf{k}}^{\prime}\right)$ if and only if it has properties 1$), 2$ ) and the following property:

3) $t_{1}(u)=t_{1}(v)$.

It is easy to deduce from this observation that in the set of identities $I\left(\mathbf{W}_{\mathbf{k}}^{\prime}\right)$, an identity of the smallest lexicographic length in which a linear word is equal to some non-linear word is an identity of the form

$$
x_{1} \ldots x_{k}=x_{1} \ldots x_{k}^{2} .
$$

Therefore the identity (3.17) is true in the variety $\mathbf{W}_{\mathbf{k}}^{\prime}$ but false in $\mathbf{W}_{\mathbf{k}+\mathbf{1}}^{\prime}$. Consequently, the inclusion of varieties $\mathbf{W}_{\mathbf{k}}^{\prime} \subseteq \mathbf{W}_{\mathbf{k}+\mathbf{1}}^{\prime}$ is strict and the lattice interval $\mathcal{I}\left(\mathbf{S l}, \mathbf{R}_{\mathbf{1}}\right)$ is infinite.

Similarly, to prove that the lattice interval $\mathcal{I}\left(\mathbf{S l}, \mathbf{A}_{\mathbf{n}}\right)$ is infinite we note that for the varieties $\mathbf{U}_{\mathbf{k}}=\mathbf{W}_{\mathbf{k}} \vee \mathbf{A}_{\mathbf{p}}$ the equality $I\left(\mathbf{U}_{\mathbf{k}}\right)=I\left(\mathbf{W}_{\mathbf{k}}\right) \cap I\left(\mathbf{A}_{\mathbf{p}}\right)$ holds. Hence we can observe that an identity $u=v$ belongs to the set of identities $I\left(\mathbf{U}_{\mathbf{k}}\right)$ if and only if it has properties 1), 2) and the property

4) for any letter $x$ we have the congruence $|u|_{x}-|v|_{x} \equiv 0(\bmod p)$.

It is easy to deduce from this observation that in the set of identities $I\left(\mathbf{U}_{\mathbf{k}}\right)$, an identity of the smallest lexicographic length in which a linear word is equal to some non-linear word is an identity of the form

$$
x_{1} \ldots x_{k}=x_{1} \ldots x_{k}^{1+p} .
$$

Therefore identity (3.18) is true in the variety $\mathbf{U}_{\mathbf{k}}$ but false in $\mathbf{U}_{\mathbf{k}+\mathbf{1}}$. Consequently, the inclusion of varieties $\mathbf{U}_{\mathbf{k}} \subseteq \mathbf{U}_{\mathbf{k}+\mathbf{1}}$ is strict and the lattice interval $\mathcal{I}\left(\mathbf{S l}, \mathbf{A}_{\mathbf{n}}\right)$ is infinite. 
To complete the proof of Theorems 3.1 and 3.2 we consider the case of the wreath product of group varieties. If we consider the wreath product of different group varieties $\mathbf{A}_{\mathbf{p}}$ and $\mathbf{A}_{\mathbf{q}}$, then the description of the lattice $\mathcal{L}\left(\mathbf{A}_{\mathbf{p}} \mathbf{w} \mathbf{A}_{\mathbf{q}}\right)$ follows from the more general Higman theorem (see [6, Theorem 54.41] or [26]), and this lattice consists of exactly 5 elements.

In the case where the group varieties involved in the wreath product are the same, we can note that the variety $\mathbf{A}_{\mathbf{p}}^{\mathbf{2}}$ is metabelian and, consequently, has a finite basis of identities [6, Theorem 36.11]. On the other hand, by Shmel'kin's theorem, this variety is not generated by any finite group [6, Theorem 24.64]. This variety contains the infinite p-group

$$
G_{\infty}=C(p) w\left(\prod_{i=1}^{\infty} C_{i}(p)\right),
$$

where $C_{i}(p) \cong C(p)$, which has trivial centre by Theorem 24.23 in [6]. Consequently, the variety $\mathbf{W}=\mathbf{A}_{\mathbf{p}}^{\mathbf{2}}$ is not a nilpotent variety. Furthermore, in $\mathcal{I}(\mathbf{W})$ there exists an infinite chain of subvarieties, since, according to the results in [29, Theorem 5.1], the nilpotency class of the wreath product of groups

$$
G_{m}=C(p) w\left(\prod_{i=1}^{m} C_{i}(p)\right)
$$

where $C_{i}(p) \cong C(p)$, is equal to $l=m(p-1)+1$. Thus, the lattice interval $\mathcal{I}(\mathbf{W})$ contains the infinite chain of varieties

$$
\mathbf{W}_{\mathbf{1}}=\mathbf{W} \cap \mathbf{N}_{1} \subset \mathbf{W} \cap \mathbf{N}_{\mathbf{2}} \subset \cdots \subset \mathbf{W} \cap \mathbf{N}_{\mathbf{k}} \subset \cdots \subset \mathbf{W},
$$

where $\mathbf{N}_{\mathbf{k}}$ is the variety of nilpotent groups of class $k$. Theorems 3.1 and 3.2 are proved.

Remark 3.19. In [1] it is noted that for $p=2$ the chain (3.20) in fact gives the entire lattice of subvarieties in $\mathcal{L}\left(\mathbf{A}_{\mathbf{2}}^{\mathbf{2}}\right)$.

Remark 3.20. We do not have complete information about the structure of the lattice $\mathcal{L}\left(\mathbf{S l w} \mathbf{N}_{\mathbf{2}}\right)$. But it is interesting to point out that this lattice contains substantially more elements than in all other cases of a finite lattice of the wreath product of atoms of semigroup varieties. Namely, the following assertion holds.

The lattice $\mathcal{L}\left(\mathbf{S l w} \mathbf{N}_{\mathbf{2}}\right)$ of all subvarieties of the variety $\mathbf{S l w} \mathbf{N}_{\mathbf{2}}$ contains at least 33 elements. The lattice interval $\mathcal{I}\left(\mathbf{S} \mathbf{1}, \mathbf{N}_{\mathbf{2}}\right)$ contains at least 18 elements.

The proof of this assertion would take up a lot of space and we do not give it here.

\section{Generating of the Wreath product of atoms By a finite semigroup}

In this section we prove the following theorem.

Theorem 4.1. The monoid wreath product $\mathbf{U w V}$ of atoms of the lattice $\mathcal{L}$ of all semigroup varieties is not generated by a finite semigroup if and only if $\mathbf{U}=\mathbf{V}=\mathbf{A}_{\mathbf{p}}$ for some prime $p$.

First of all we note that a large part of the theorem already follows from the preceding results of this paper. We begin with the following well-known and simple remark.

Remark 4.2. The lattice join of varieties $\mathbf{U} \vee \mathbf{V}$ is generated by the direct product $S \times R$, where $\mathbf{U}=\operatorname{var} S$ and $\mathbf{V}=\operatorname{var} R$. 
This remark and the results of $\S 2$ imply that quite a number of monoid wreath products of atoms of the lattice of semigroup varieties are generated by a finite semigroup. Furthermore, results on generation of the monoid wreath product of atoms of the lattice of semigroup varieties are contained in Theorem 6.1 in [10, Proposition 7.2 in [12, Corollaries 2.18, 2.26 (or Lemma 3.18), and Proposition 2.30. Thus, to complete the proof of Theorem 4.1 it remains to consider the cases of the monoid wreath products $\mathbf{R}_{1} \mathbf{w N}_{2}, \mathbf{R}_{1} \mathrm{wR}_{1}, \mathbf{R}_{1} \mathrm{wSl}$, SlwN $\mathbf{N}_{2}$, Slw $\mathbf{L}_{1}, \mathbf{A}_{\mathrm{p}} \mathbf{w N}_{2}$, and the case of the wreath product of group atoms. Furthermore, in the case of group atoms, the result of the theorem easily follows from Shmel'kin's well-known and beautiful theorem [6, Theorem 24.64] (see also [18, 19]), which gives necessary and sufficient conditions for a product of group varieties to be generated by a finite group.

Proposition 4.3. The monoid wreath product $\mathbf{R}_{\mathbf{1}} \mathbf{w} \mathbf{N}_{\mathbf{2}}$ is generated as a variety by the four-element semigroup $T_{1}=\left\{e, a, a^{2}, 0\right\}$ obtained from the monogenic nilpotent threeelement semigroup $\left\langle a \mid a^{3}=0\right\rangle$ by adjoining an element $e$ such that ae $=e^{2}=e$ and $e a=0$.

Proof. According to Corollary 2.4, the identity

$$
y_{1} y_{2} x=z_{1} z_{2} x
$$

is a basis of identities of the variety $\mathbf{R}_{\mathbf{1}} \mathbf{w} \mathbf{N}_{\mathbf{2}}$. We now show that the semigroup $T_{1}$ has the same basis of identities. Indeed, for $x=e$ we have $y_{1} y_{2} e=e=z_{1} z_{2} e$, and for $x=a$ we have $y_{1} y_{2} a=0=z_{1} z_{2} a$ for any values of $y_{1}$ and $y_{2}$. If an identity $u=v$ does not belong to the set $I\left(\mathbf{R}_{\mathbf{1}} \mathbf{w} \mathbf{N}_{\mathbf{2}}\right)$, then either $t_{1}(u) \neq t_{1}(v)$ or the length of at least one of the words $|u|,|v|$ is less than 3 . In the first case, in the identity $u_{1} z=v_{1} t$, where $z \neq t$, we set $z=0, t=e$. Then we obtain that the value of the word $u$ is equal to 0 , while the value of the word $v$ is equal to $e$. In the second case we can assume that $|u|=2$ and $|v| \geq 2$ and, moreover, $u \equiv z^{2}$ and $v \equiv v_{1} z$. Setting $z=a$ and setting the values of the other variables to be equal to $e$, we obtain that the value of the word $u$ is equal to $a^{2}$, while the value of the word $v$ is equal to 0 , provided these words are different.

Proposition 4.4. The monoid wreath product $\mathbf{R}_{\mathbf{1}} \mathbf{w} \mathbf{R}_{\mathbf{1}}$ is generated as a variety by the four-element semigroup $T_{2}=\left\{b_{1}, b_{2}, b_{3}, d\right\}$ in which $x b_{i}=b_{i}(i=1,2,3)$ for any $x \in T_{2}$, and the other products are $b_{3} d=b_{2}, b_{2} d=b_{1} d=d^{2}=b_{1}$.

Proof. It is easy to verify that the groupoid $T_{2}$ defined in Proposition 4.4 is associative. According to Corollary 2.4, the variety $\mathbf{R}_{\mathbf{1}} \mathbf{w} \mathbf{R}_{\mathbf{1}}$ has a basis of identities consisting of the single identity

$$
x y_{1} y_{2}=y_{1} y_{2} .
$$

Obviously, $T_{2}^{2}$ coincides with the ideal $R=\left\{b_{1}, b_{2}, b_{3}\right\}$ of right zeros in $T_{2}$. Therefore the identity (4.2) is true in $T_{2}$. Conversely, since the semigroup $T_{2}$ has right zeros, we have $t_{1}(u)=t_{1}(v)$. If in addition $t_{2}(u) \neq t_{2}(v)$, then $T_{2}$ satisfies an identity of the form $z x=y x$, where $y \neq z$. Setting $x=z=d$ and $y=b_{3}$ we obtain that $x z=d^{2}=b_{1}$, while $y x=b_{3} d=b_{2}$. Consequently, any identity that does not belong to $I\left(\mathbf{R}_{\mathbf{1}} \mathbf{w} \mathbf{R}_{\mathbf{1}}\right)$ is false in $T_{2}$.

Proposition 4.5. The monoid wreath product $\mathbf{S l w N}_{2}$ is generated as a variety by the sixteen-element semigroup $T_{0}=U_{2} w N$, where $U_{2}=\{0,1\}$ is the two-element semilattice and $N=\{0, a\}$ is the two-element nilpotent semigroup.

Proof. Recall that the description of identities of the variety $\mathrm{SlwN}_{2}$ was given in Corollary 2.13. By using Remark 2.1 it is easy to establish that the wreath product $T_{0}=U_{2} w N$ satisfies the same identities as the variety $\mathrm{SlwN}_{\mathbf{2}}$. 
Proposition 4.6. The monoid wreath product $\mathbf{S l w}_{\mathbf{1}}$ is generated as a variety by the four-element semigroup $T^{\prime}=\left\{e, a, a^{2}, b\right\}$ in which $e$ is a right identity element, that is, $x e=x$ for any $x$, the elements $a^{2}, b$ are left zeros, and $e y=b$, ay $=a^{2}$ for $y \in\left\{a, a^{2}, b\right\}$.

Proof. By Lemma 2.10, the set consisting of the two identities

$$
\begin{aligned}
z y & =z y^{2}, \\
z y x & =z x y
\end{aligned}
$$

is a basis of identities of the variety $\mathbf{S l w L}_{\mathbf{1}}$. It is easy to verify that the identities (4.3) and (4.4) are true in the semigroup $T^{\prime}$. Using Proposition 3.6 we can assert that if $\operatorname{var} T^{\prime} \neq \mathbf{S l w L}_{\mathbf{1}}$, then either $\operatorname{var} T^{\prime} \subseteq \mathbf{V}=\mathbf{S l} \vee \mathbf{L}_{\mathbf{1}} \vee \mathbf{N}_{\mathbf{2}}$ and, as is well known, the latter variety has a basis consisting of the identities (4.3), (4.4) and the identity

$$
z x=z^{2} x,
$$

or $\operatorname{var} T^{\prime} \subseteq \operatorname{var} P^{\prime}$ and, as is well known, the variety var $P^{\prime}$ has a basis of identities consisting of the identity (4.3) and the identity

$$
x^{2} y=y^{2} x .
$$

It remains to observe that the identity (4.5) is false in $T_{0}$ if we set $z=a$ and $x=e$, while the identity (4.6) is false in $T_{0}$ if we set $x=a$ and $y=b$. Consequently, $T^{\prime} \notin \mathbf{V}$ and $T^{\prime} \notin \operatorname{var} P^{\prime}$. Thus, $\operatorname{var} T^{\prime}=\mathbf{S l w}_{\mathbf{1}}$.

Corollary 4.7. The monoid wreath product $\mathbf{R}_{\mathbf{1}} \mathrm{wSl}$ is generated as a variety by a fourelement semigroup.

Proof. This follows from the duality of the bases of identities of the varieties $\mathbf{R}_{\mathbf{1}} \mathbf{w S l}$ and $\mathbf{S l w L}_{\mathbf{1}}$ and from Proposition 4.6.

To find a semigroup generating the variety $\mathbf{A}_{\mathbf{p}} \mathbf{w} \mathbf{N}_{2}$, we consider the extended standard wreath product $T_{p}^{\prime}=C(p) w N$ in which $N=\{a, 0\}$ is the two-element semigroup with zero multiplication and $C(p)$ is the cyclic group of order $p$ with a generator $d$. In the wreath product $T_{p}^{\prime}$ we consider the subsemigroup $T_{p}=\left\langle(f, a),\left(c_{d}, 0\right)\right\rangle$ generated by two elements. Here $c_{d}$ is the constant function that is identically equal to the element $d$ of the group, and the function $f$ is defined by the equalities $f(1)=f(a)=e, f(0)=d$. It is easy to verify that ${ }^{0} f=c_{d}$ and ${ }^{a} f(1)=e,{ }^{a} f(0)={ }^{a} f(a)=d$. Next, calculating the powers of the element $(f, a)$ we obtain

$$
\begin{gathered}
(f, a)^{2}=\left(f^{a} f, 0\right), \quad(f, a)^{3}=\left(f^{a} f c_{d}, 0\right), \\
(f, a)^{4}=\left(f^{a} f c_{d}^{2}, 0\right), \quad \ldots, \quad(f, a)^{2+p}=\left(f^{a} f, 0\right)=(f, a)^{2} .
\end{gathered}
$$

Thus, the monogenic semigroup generated by the element $(f, a)$ contains $p+1$ elements. The monogenic semigroup generated by the element $\left(c_{d}, 0\right)$ is a cyclic group of order $p$. Furthermore, $(f, a)\left(c_{d}^{i}, 0\right)=\left(f c_{d}^{i}, 0\right)$ and $\left(c_{d}^{i}, 0\right)(f, a)=\left(c_{d}^{i+1}, 0\right)$. Thus, we have $f c_{d}^{i}(1)=$ $f c_{d}^{i}(a)=d^{i}, \quad f c_{d}^{i}(0)=d^{i+1}$, and $f^{a} f c_{d}^{i}(1)=d^{i}, \quad f^{a} f c_{d}^{i}(a)=d^{i+1}, \quad f^{a} f c_{d}^{i}(0)=d^{i+2}$. Consequently, the semigroup $T_{p}$ contains $3 p+1$ elements: namely, $(f, a),\left(c_{d}^{i}, 0\right),\left(f c_{d}^{i}, 0\right)$, $\left(f^{a} f c_{d}^{i}, 0\right) \quad(i=1, \ldots, p)$.

Proposition 4.8. The monoid wreath product $\mathbf{A}_{\mathbf{p}} \mathbf{w} \mathbf{N}_{\mathbf{2}}$ is generated as a variety by the semigroup $T_{p}$ defined above.

Proof. By the construction of the semigroup $T_{p}$ it belongs to $\mathbf{A}_{\mathbf{p}} \mathbf{w} \mathbf{N}_{\mathbf{2}}$. By Proposition 3.12, if $\operatorname{var} T_{p} \neq \mathbf{A}_{\mathbf{p}} \mathbf{w} \mathbf{N}_{\mathbf{2}}$, then either $T_{p} \in \mathbf{L}_{\mathbf{2}, \mathbf{2}}$ or $T_{p} \in \mathbf{U}_{\mathbf{p}}=\mathbf{A}_{\mathbf{p}} \vee \mathbf{L}_{\mathbf{1}} \vee \mathbf{N}_{\mathbf{2}}$. Obviously, $T_{p} \notin \mathbf{L}_{\mathbf{2}, \mathbf{2}}$, since $T_{p}$ contains a group of order $p$. On the other hand, $T_{p} \notin \mathbf{U}_{\mathbf{p}}$, since $T_{p}$ does not satisfy the identity (4.4), which is true in $\mathbf{U}_{\mathbf{p}}$. Indeed, setting $z=y=(f, a), \quad x=\left(c_{d}, 0\right)$ we obtain that $z y x=\left(f^{a} f c_{d}, 0\right)$ and $z x y=\left(f c_{d}^{2}, 0\right)$. 
Here, $f^{a} f c_{d}(1)=d$, while $f c_{d}^{2}(1)=d^{2}$, that is, the identity (4.4) is false in $T_{p}$. Consequently, $\operatorname{var} T_{p}=\mathbf{A}_{\mathbf{p}} \mathbf{w} \mathbf{N}_{\mathbf{2}}$ for any $p \geq 2$.

The proof of Proposition 4.8 completes the proof of Theorem 4.1.

\section{REFERENCES}

[1] V. A. Artamonov, Chain varieties of groups, Trudy Sem. Petrovsk. 1978 (1978), no. 3, 3-8. (Russian) MR0494776 (80m:20027)

[2] A. P. Biryukov, Varieties of idempotent semigroups, Algebra i Logika 9 (1970), 255-273; English transl. in Algebra and Logic 9 (1971), 153-164. MR0297897 (45:6949)

[3] É. A. Golubov and M. V. Sapir, Varieties of finitely approximable semigroups, Dokl. Akad. Nauk SSSR 247 (1979), 1037-1041; English transl. in Soviet Math. Dokl. 20 (1979), 828-832. MR0550461 (81d:20050)

[4] É. A. Golubov and M. V. Sapir, Varieties of finitely approximable semigroups, Izv. Vyssh. Uchebn. Zaved. Mat. 1982, no. 11, 21-29; English transl. in Sov. Math. 26 (1982), no. 11, 25-36. MR0687309 (85f:20051)

[5] G. Lallement, Semigroups and combinatorial applications, John Wiley and Sons, New York, 1979. MR0530552 (81j:20082)

[6] H. Neumann, Varieties of groups, Springer-Verlag, New York, 1967. MR0215899 (35:6734)

[7] V. V. Rasin, Free completely simple semigroups, Ural. Gos. Univ. Mat. Zap. 11 (1979), no. 3, 140-151. (Russian) MR0573889 (81i:20079a)

[8] A. V. Tishchenko, A remark on semigroup varieties of finite index, Izv. Vyssh. Uchebn. Zaved. Mat. 1990, no. 7, 79-83; English transl. in Soviet Math. (Iz. VUZ) 34 (1990), no. 7, 92-96. MR1104152 (92h:20087)

[9] A. V. Tishchenko, When does the monoid wreath product of varieties of semigroups have finite index?, Int. Conf. on Algebra (Barnaul, August 1991), Abstracts of talks, Novosibirsk, 1991, p. 144. (Russian)

[10] A. V. Tishchenko, On different definitions of the wreath product of semigroup varieties, Fundam. Prikl. Mat. 2, no. 2 (1996), 233-249. (Russian) MR1789007

[11] A. V. Tishchenko, The monoid of semigroup varieties with respect to a wreath product, Uspekhi Mat. Nauk 51 (1996), no. 2, 177-178; English transl. in Russian Math. Surveys 51 (1996), 351-352. MR 1401556

[12] A. V. Tishchenko, Wreath products of varieties, and semi-Archimedean varieties of semigroups, Trudy Mosk. Mat. Obshch. 57 (1996), 218-238; English transl. in Trans. Moscow Math. Soc. 1996, 203-222. MR 1468982 (99d:20101)

[13] A. V. Tishchenko, The wreath product of the atoms of the lattice of semigroup varieties, Uspekhi Mat. Nauk 53 (1998), no. 4, 219-220; English transl. in Russian Math. Surveys 53 (1998), 870-871. MR 1668074

[14] A. V. Tishchenko, On the lattice of subvarieties of the monoid wreath product of varieties, 2-nd Int. Conf. "Semigroups: theory and applications" (St.-Petersburg, 1999), Abstracts of talks, St. Petersburg, 1999, pp. 98-99. (Russian)

[15] A. V. Tishchenko, The ordered monoid of semigroup varieties with respect to a wreath product, Fundam. Prikl. Mat. 5 (1999), no. 1, 283-305. (Russian) MR1799534 (2001k:20125)

[16] L. N. Shevrin and M. V. Volkov, Identities of semigroups, Izv. Vyssh. Uchebn. Zaved. Mat. 1985, no. 11, 3-47; English transl. in Sov. Math. 29 (1985), no. 11, 1-64. MR0829099 (87f:20094)

[17] L. N. Shevrin and E. V. Sukhanov, Structural aspects of the theory of varieties of semigroups, Izv. Vyssh. Uchebn. Zaved. Mat. 1989, no. 6, 3-39; English transl. in Soviet Math. (Iz. VUZ) 33 (1989), no. 6, 1-34. MR1017775 (91a:20071)

[18] A. L. Shmel'kin, Wreath products and varieties of groups, Dokl. Akad. Nauk SSSR 157 (1964), 1063-1065. (Russian) MR0166262 (29:3539)

[19] A. L. Shmel'kin, Wreath products and varieties of groups, Izv. Akad. Nauk SSSR Ser. Mat. 29 (1965), 149-170. (Russian) MR0193131(33:1352)

[20] J. Almeida, Semidirect products of pseudovarieties from the universal algebraist's point of view, J. Pure Appl. Algebra 60 (1989), 113-128. MR1020712 (91a:20068)

[21] J. Almeida, Semidirectly closed pseudovarieties of locally trivial semigroups, Semigroup Forum 40 (1990), 315-323. MR1038009 (91a:20070)

[22] J. Almeida, On iterated semidirect products of finite semilattices, J. Algebra 142 (1991), 239-254. MR.1125216 (92f:20061)

[23] S. Eilenberg, Automata, Languages, and Machines. Vol. B, Academic Press, New York, 1976. MR 0530383 (58:26604a) 
[24] S. Eilenberg and M. P. Schutzenberger, On pseudovarieties, Advances Math. 19 (1976), 413-418. MR0401604 (53:5431)

[25] T. Evans, The lattice of semigroup varieties, Semigroup Forum 2 (1971), 1-43. MR0284528 $(44: 1753)$

[26] G. Higman, Some remarks on varieties of groups, Quart. J. Math. Oxford (2) 10 (1959), 165-178. MR0113925 (22:4756)

[27] C. Irastorza, Base non finie de variétés, Proc. STACS 85 (Saarbrc̈ken, 1985), Lecture Notes in Comput. Sci. 182, Springer-Verlag, Berlin, 1985, pp. 180-186. MR0786881 (86j:68068)

[28] Yu. G. Koshelev, Varieties preserved under wreath products, Semigroup Forum 12 (1976), 95-107. MR0399330 (53:3175)

[29] H. Liebeck, Concerning nilpotent wreath products, Proc. Cambridge Phil. Soc. 58 (1962), 443-451. MR0139656 (25:3087)

[30] J.-E. Pin, On semidirect products of two finite semilattices, Semigroup Forum 28 (1984), 73-81. MR0729653 (85b:20049)

[31] V. V. Rasin, On the lattice of varieties of completely simple semigroups, Semigroup Forum 17 (1979), 113-122. MR0527213(80b:20073)

[32] V. V. Rasin, On the varieties of Cliffordian semigroups, Semigroup Forum 23 (1981), 201-220. MR0647112 (83c:20073)

[33] B. Tilson, Categories as algebra: an essential ingredient in the theory of monoids, J. Pure and Appl. Algebra 48 (1987), 83-198. MR0915990(90e:20061)

[34] A. V. Tishchenko, Wreath products of atoms of the lattice of semigroup varieties, Int. Conf. "Semigroups and their applications including semigroup rings" (St. Petersburg, 1995), Abstracts of talks, St. Petersburg, 1995, pp. 72-73. (Russian)

Finance Academy at the Government of the Russian Federation and Moscow State Humanitarian Boarding Institute, Moscow, Russia

Translated by E. KHUKHRO 